\title{
1 Reshaping the Hexagone: the genetic landscape of modern
}

\section{France}

4 Simone Andrea Biagini ${ }^{1}$, Eva Ramos-Luis ${ }^{2,3}$, David Comas ${ }^{1}$, Francesc Calafell ${ }^{1 *}$

\section{Abstract}

Unlike other European countries, the human population genetics and demographic history of Metropolitan France is surprisingly understudied. In this work, we combined newly genotyped samples from various zones in France with publicly available data and applied both allele frequency and haplotype-based methods in order to describe the internal structure of this country, by using genome-wide single nucleotide polymorphism (SNP) array genotypes. We found out that French Basques are genetically distinct from all other populations in the Hexagone and that the populations from southwest France (namely the Gascony region) share a large proportion of their ancestry with Basques. Otherwise, the genetic makeup of the French population is relatively homogeneous and mostly related to Southern and Central European groups. However, a fine-grained, haplotype-based analysis revealed that Bretons slightly separated from the rest of the groups, due mostly to gene flow from the British Isles in a time frame that coincides both historically attested Celtic population movements to this area between the 3 th and the 9th centuries CE, but also with a more ancient genetic continuity between Brittany and the British Isles related to the shared drift with huntergatherer populations. Haplotype-based methods also unveiled subtle internal structures and connections with the surrounding modern populations, particularly in the periphery of the Hexagone. 


\section{Introduction}

44 Located in the center of Western Europe, Metropolitan France has historically acted as a

45 bridge connecting Northern Europe to the Mediterranean and the Iberian spaces. The 46 geographical position of France strongly affected the history of the settlement of the

47 different parts of the territory, whose continuous fragmentation through time is attested

48 by the large number of populations and cultures that settled this area. Greeks, Romans

49 and Celtic tribes from central Europe shaped a first internal structure between the 6th

50 and the 1st centuries BCE, while waves of barbarian invasions (Alamanni, Burgundians,

51 Visigoths, Franks, and Celts) strongly impacted the population landscape of France

52 during the 5 th century $\mathrm{CE}^{1}$. During the 9 th and 10 th centuries $\mathrm{CE}$, foreign invasions

53 from all sides also influenced the territory: Muslims and Saracens from North Africa

54 coming through Iberia, Hungarian Magyar from the east, and Vikings (Northmen) from

55 the north ${ }^{1}$. Nowadays, France is a cosmopolitan country whose society is shaped by a

56 plurality of lifestyles and truly different ethno-cultural diversity. Without any doubt,

57 the impact of political refugees throughout the 20th century, or of the immigration

58 from colonized countries to mainland France, such as the migration of Arabs and

59 Berbers from Algeria which was the most extensive of all colonial migrations to

60 Western Europe before the 1960s ${ }^{2}$, enriched the modern genetic landscape of the

61 French territory. However, it is beyond our intention to explore this plethora of recent

62 genetic contributions here, which can be quantified much more precisely with

63 demographic analyses. Instead, we can apply genomic tools to excavate a deeper and

64 ancient genetic background.

65 At the light of this complex past, the genetic landscape of France has been poorly

66 analyzed, especially in recent times. The first studies with classical markers defined a

67 general heterogeneous pattern considering different geographical arrangements such as

68 military districts, historical provinces, and regions ${ }^{3,4}$. With his synthetic maps, Cavalli-

69 Sforza proposed that this heterogeneity was a consequence of differential Neolithic

70 influences between northern and southern France, and also pointed out a differentiation

71 for Brittany and Gascony ${ }^{5}$. More recently, studies on mitochondrial DNA highlighted a

72 general homogeneity when the samples were distributed among the 22 regions

73 established in 1982 and historic provinces ${ }^{6,7}$. Generally, the mtDNA haplogroup 
composition of French people did not differentiate neither internally, nor from the surrounding European genetic landscape ${ }^{6,7}$. On a microgeographical scale, Brittany showed affinity with Scandinavia and Britain, while French Basques stood out for a high frequency of haplogroup $\mathrm{H}$, suggesting a link with the Neolithic diffusion in Europe ${ }^{6,7}$. In agreement with the homogeneity described by mtDNA studies, the Ychromosome diversity strongly pointed out a lack of differentiation between the distinct groups when samples were organized on a regional scale. Even in this case, Brittany represented an exception, showing a lower Y-chromosome diversity that was interpreted as consequence of a possible founder effect, plus an isolation process ${ }^{8}$. Based on autosomal variants, a genome-wide study on Western France did not find any differentiation among the distinct groups organized on a regional geographical distribution ${ }^{9}$. Even in this case, the only outlier was Brittany, whose higher linkage disequilibrium suggested a lower effective population size, thus supporting the hypothesis of isolation inferred by the outcomes of the Y-chromosome analyses. Furthermore, in agreement with mitochondrial studies, Bretons were found to be admixed with individuals from the British Isles ${ }^{9}$. In this work, we present a comprehensive genome-wide study on France, using both allele frequency and haplotype-based methods, to determine the minimal meaningful geographic unit of genetic differentiation within France, describe the geogenetical landscape patterns within France, and trace the historic and ancient sources of gene flow into the Hexagone.

5

\section{Material and Methods}

\section{Dataset arrangement and genotypes}

In this study, informed consent was obtained from 331 individuals from different French departments. Internal Review Board approval for this work was granted by CEIC-PSMAR ref. 2016/6723/I. These samples were compiled by the Institute of Forensic Sciences, University of Santiago de Compostela, and most of them were first reported in an analysis of Y-chromosome markers in ref. ${ }^{8}$. As specified in the latter work, all the subjects and their parents were born in mainland France and bore a French surname. DNA was extracted from blood samples as described in Ramos-Luis et al. ${ }^{8}$. A 
107 total of four Axiom ${ }^{\circledR}$ Genome-Wide Human Origins Arrays ( $\left.\sim 29 \mathrm{~K} \mathrm{SNPs}\right){ }^{10}$ were

108 genotyped at the Centro Nacional de Genotipado - Universidade de Santiago de

109 Compostela facility. Genotype calling was performed running four different batches

110 according to the Affymetrix Best Practices Workflow implemented in the software

111 Axiom $^{\mathrm{TM}}$ Analysis Suite 2.0. Out of 331 samples, 52 failed the genotyping process and

112 a total of 279 samples were retained. Three additional samples were removed following

113 an Identity-by-descent analysis (IBD) since they displayed a Proportion IBD value $\geq$

1140.125 (minimum threshold for removing relatedness equal or higher than a third

115 degree). Eventually, 276 samples were retained. To complete the French dataset, 79

116 additional samples from a public source ${ }^{11}$ and 60 from unpublished data (from an

117 ongoing study on the Basque Country and the Franco-Cantabrian region; samples are

118 subset from those in ref. ${ }^{12}$ ) were added to the original 276 , leading to a total of 415

119 samples. In a preliminary part of this work, 20 out of the 276 samples were identified as

120 outliers and removed from the study (see Supplementary Figure 1 and caption). Thus,

121 the complete dataset included 256 newly genotyped samples, plus 139 additional ones,

122 for a final group of 395 samples (Dataset A) distributed among 20 different French

123 departments (see Supplementary Figure 2 for the geographical distribution). For the

124 allele frequency analyses, as comparison with external populations, a total of 333

125 samples were added to Dataset A, forming Dataset B. This external group included 218

126 samples among Germany, Norway, Spain, Italy, England, Ireland, and Scotland ${ }^{11}$,

127 together with 107 samples from the Spanish autonomous communities of Catalonia,

128 Valencian Community, and Balearic islands ${ }^{13}$, and 8 additional samples from South

129 Italy (Naples) newly genotyped with Axiom ${ }^{\circledR}$ Genome-Wide Human Origins Arrays

130 ( 629 K SNPs). Further 799 samples from external populations ${ }^{11}$ were added to the

131 previous ones when applying haplotype-based methods (Dataset C). Lastly, in the

132 analysis with ancient data, 282 ancient samples ${ }^{11}$ were added to the previous dataset,

133 with the only exclusion of the 122 sub-Saharan African samples (Dataset D) since their

134 presence would have reduced the resolution for the distribution of the rest of the

135 samples in the PCA, masking signals of admixture in the dedicated analyses (see

136 Supplementary Figure 3 for the geographical distribution of the modern samples from

137 Datasets B and C, and Supplementary table 1 for a summary of the different dataset

138 composition).

139

140 Data Quality Control 
142 Data were prepared using PLINK $1.9^{14}$. Uniparental markers and X-chromosome

143 variants were excluded. For the French dataset, a preliminary set of filters were applied

144 to each group separately before the merging process. We filtered out all variants with

145 missing call rates greater than 5\%, those that failed Hardy-Weinberg test at $\mathrm{p}<10^{-5}$,

146 and samples with more than $10 \%$ missing genotype data. After merging, only variants

147 common to the three datasets were retained and SNPs with a minor allele frequency

148 (MAF) below 5\% were excluded, resulting in a final 343,884 variants used for

149 haplotype-based methods (Dataset A). For the analyses that needed a set of independent

150 markers, SNPs were pruned setting a pairwise linkage disequilibrium maximum

151 threshold of 0.5, a window of size 200 and a shift step of 25. Eventually, the pruned

152 data retained 142,803 variants (Dataset A). In the analyses that included the external

153 populations, only the pruned dataset, consisting in 154,889 SNPs, was used for the

154 allele frequency analyses (Dataset B), while a set of 380,697 variants was retained in the

155 haplotype-based methods (Dataset C). Regarding Dataset D, a set of 163,631 SNPs was

156 retrieved after pruning (See Supplementary table 1 for a summary).

\section{Statistical analyses}

160 Eigenvectors were computed using the SmartPCA program in Eigenstrat software 161 package (v. 13050) ${ }^{15}$. For Dataset D, we used the option lsqproject:YES when

162 projecting ancient on top of the modern samples. Results were plotted in R (v 3.0.1).

164 The $F_{S T}$ fixation index was computed using the SmartPCA tool (v. 13050) from the

165 Eigenstrat software package. Results were produced in Rstudio ${ }^{16}$ using R version 3.4.4

$166{ }^{17}$. The $F_{S T}$ matrix was used together with a geographic distance matrix produced with

167 The Geographic Distance Matrix Generator (v. 1.2.3, available from

168 http://biodiversityinformatics.amnh.org/open_source/gdmg) in order to perform a

169 Mantel test correlation using the ade $4{ }^{18}$ library in R. Results were displayed using

170 ggplot2 ${ }^{19}$ and reshape ${ }^{20}$ libraries.

172 Based on different hierarchical levels (within Departments, Between Departments

173 within Areas/Regions, Between Areas/Regions; see Supplementary Figure 4 for a visual

174 representation of the used Areas and Regions), AMOVA was performed using the 


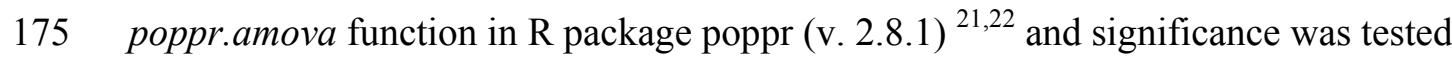

176 with the randtest function implemented in R package ade4. For every percentage of

177 variance, a p-value was calculated based on 1000 permutations.

178 Patterns of population structure were explored, in both Dataset B and D, using

179 ADMIXTURE ${ }^{23}$ testing from $\mathrm{K}=2$ to $\mathrm{K}=10$ ancestral clusters and using 10 independent

180 random seeds. Results were represented using the software pong ${ }^{24}$. For Dataset B,

181 admixture was formally tested with $\mathrm{f} 3$ statistics computed using the $q p 3 P o p$ function

182 implemented in Admixtools ${ }^{10}$, while outgroup- $f 3$ statistics were tested for Dataset $\mathrm{D}$ in

183 the form of $\mathrm{f} 3$ (Ancient, X; Mbuti), where 3 Mbuti samples from ref. ${ }^{11}$ were added to

184 Dataset D (1690 total samples, same variants as in Dataset D).

\section{EEMS (Estimated Effective Migration Surface)}

EEMS $^{25}$ analysis was run using Dataset A (142,803 variants from the pruned file).

189 With a matrix of average pairwise genetic dissimilarities calculated using the internal

190 program bed2diffs, a sample coordinates file, and a habitat coordinates file generated

191 using Google Earth Pro (v. 7.3.2.5495), we performed 10 pilot runs of 6 million MCMC

192 iterations each, with 3 million burn-in, and a thinning interval of 30,000. A second set

193 of 5 runs was then performed restarting the chain with the highest likelihood with 4

194 million MCMC iterations, 1 million burn-in, and thinning interval of 10,000. The

195 density of the population grid was set to 300 demes, and random seeds were used for

196 each one of the runs. We used the default hyperparameter values but tuned some of the

197 proposed variances to improve convergence in the second set of runs. Results for the

198 chain with the highest likelihood were displayed using eems.plots function in the $\mathrm{R}$

199 package rEEMSplots.

\section{Haplotype-based analysis}

Two different analyses were performed: one on the internal French population only

204 (Dataset A), and one also including external populations (Dataset C). In both cases,

205 phasing was performed using the software Shapeit (v. v2.r837) ${ }^{26,27}$. When running

206 ChromoPainter ${ }^{28}$, all samples were used as both recipients and donors, ${ }^{28}$ without any

207 population specification (-a option) and not allowing self-copying. First, the parameters

208 for the switch rate and global mutation probability were estimated with the EM 
209 algorithm implemented in ChromoPainter using the parameters -i 15 -in -iM for

210 chromosomes $1,7,14$, and 20 for all the samples. This step allows to estimate the two

211 parameters that will be then averaged for all chromosomes. The outcome for the average

212 weighted values for the global mutation probability and the switch rate parameters were

213 respectively 0.000745 and 266.67196 for Dataset A, and 0.000586 and 237.50784 for

214 Dataset C. In a second step, ChromoPainter was run for all chromosomes using the two

215 fixed parameters. Later, the final coancestry matrices for each chromosome were

216 combined using the tool Chromocombine. The latter also estimates the $\mathrm{C}$ parameter

217 which is needed for the normalization of the coancestry matrix data when we run

218 fineSTRUCTURE in order to identify the population structure. The MCMC of

219 fineSTRUCTURE was run using 1000000 burn-in iterations (flag -x), 2000000

220 iterations sampled (flag -y), and thinning interval of 10000 (flag -z). Eventually, the

221 fineSTRUCTURE tree was estimated running three different seeds and using the flags -

$222 \mathrm{X}-\mathrm{Y}-\mathrm{m} \mathrm{T}$ that allow to build the sample relationship tree. In the analysis on Dataset $\mathrm{C}$,

223 the work was then divided in two phases. In the first one, ChromoPainter and

224 fineSTRUCTURE were rerun, this time silencing France in order to define the external

225 groups only. In the second phase, fineSTRUCTURE was rerun using the "force file"

226 option (-F), using "continents" as donor groups (represented by the external groups

227 defined in the first phase); $-\mathrm{F}$ is a function that allows to exclude the donor

228 representation in the building tree phase and focus on the distribution of the recipient

229 groups, represented by the French samples only. We then applied the non-negative-

230 least-squares (nnls) function from GLOBETROTTER ${ }^{29}$ in order to describe the

231 ancestry profiles for the French groups we detected with the "force file" option. We

232 then used GLOBETROTTER in order to describe admixture events, sources and dates.

233 More details about the usage of GLOBETROTTER are reported in Supplementary note

2341.

Results

Internal genetic structure in France

240 In order to define the best geographical partitioning of genetic differentiation, a

241 hierarchical analysis of molecular variance (AMOVA) was performed with areas or 
242 regions as major grouping factors. We determined first the proportion of genetic

243 variation partitioned among geographic areas, among departments within geographic

244 areas, and within departments. We next tested the proportion of genetic variation

245 partitioned among regions (considering the 13 regions established in 2016), among

246 departments within regions, and within departments. A further AMOVA was performed

247 only testing the proportion of genetic variation partitioned among and within

248 departments. As shown in Table 1, in all cases the main contribution to the genetic

249 variance was found at the lowest hierarchical level (variation within departments), while

250 differences among regions resulted in a negative value that could be interpreted as zero,

251 meaning absence of any structure at this level. Conversely, differences among areas

252 displayed positive values, supporting the role of areas as more reliable grouping factors

253 of genetic variations when considering wider sample distributions. Finally, the results

254 for the variation between departments, also supported by significant $p$-values in all the

255 AMOVA analyses, pointed to the fact that this level of stratification might be a better

256 representation for the minimal unit of genetic differentiation. Based on these results,

257 samples were distributed on the map according to the departmental locations

258 (Supplementary Figure 2) and all the subsequent analyses considered this grouping

259 factor, although, given their known cultural and genetic identity, we retained Basque-

260 speakers as a separate group in the Pyrénéés-Atlantiques department. A first Principal

261 Component Analysis (PCA) showed two distinct groups separated along the first PC

262 (Figure 1A): the Basque samples on the right part of the plot, against most of the rest of

263 the samples on the left one, within which a structure cannot be defined. These two

264 major groups are connected by a "bridge" of samples represented by non-Basque-

265 speaking individuals from the Gascony region in the southwestern corner of France.

266 When we averaged the eigenvalues for the first two PCs and represented the same PCA,

267 together with standard deviation (SD) values for each group, no evident pattern could

268 still be discerned beyond the separation of Basques and Gascons (Supplementary Figure

269 5A). When we removed both Basque and Gascon samples from the analysis (Figure

270 1B), the resulting PCA showed some internal pattern of differentiation, more clearly

271 defined by the average PCA (Supplementary Figure 5B), in which samples from the

272 departments belonging to the northwestern region of Brittany seem to form a cluster on

273 the left part of the plot.

274

275 Patterns of gene flow within France 
277 In the genetic variation computed with the $F_{S T}$ analysis, a general homogeneous pattern

278 was found, with fine scale values of differentiation between some departments. The southwestern samples (Basques and Gascons) showed the highest values of differentiation with the northwestern departments reaching scores between 0.008 and 0.009 for the Basque-speaking samples, and between 0.004 and 0.006 for the non-

282 Basque-speaking ones (Supplementary Figure 6A, left), followed by lower values of differentiation with the northern and northeastern departments. Without the southwestern samples, the main differentiation was recorded between the northwestern departments and the southeastern corner of the country, with a highest value of differentiation around 0.002 between the southeastern department of Bouches-du-Rhône $(\mathrm{BdR})$ and the northwestern Breton department of Côtes-d'Armor (CdA) northeastern ones. A Multidimensional Scaling analysis (MDS) based on the $F_{S T}$ matrices clearly showed how the southwestern samples separate from the rest of the groups (Supplementary Figure 6A, right), and how the Breton departments do the same once the Gascon and Basque samples are removed (Supplementary Figure 6B, right). A Mantel test of isolation by distance (IBD) between the $F_{S T}$ values and the geographical distances showed a positive and statistically supported correlation $\left(\mathrm{R}^{2}=0.332, \mathrm{P}=0.001\right)$ (Supplementary Figure 7A), moving to even more positive values when the southwestern samples were removed $\left(\mathrm{R}^{2}=0.432, \mathrm{P}=0.001\right)$ (Supplementary Figure 7B).

298 Next, we used the EEMS analysis, a method for visualizing genetic diversity patterns,

299 and found that the resulting effective migration surface mirrors the outcomes of genetic

300 differentiation detected by the $F_{S T}$ analyses (Figure 2); a higher effective migration was

301 locally found in northern, northeastern and northwestern France among departments

302 belonging to the same geographical areas, while a major barrier was discovered along 303 the western side of France.

\section{Haplotype sharing patterns within France}

307 Using haplotype-based methods (Dataset A), we looked for patterns of haplotype

308 sharing, illustrating relations between departments. In this first step, cutting the

309 fineSTRUCTURE tree at the very base, allowed us to describe a fine scale haplotype 
310 sharing distribution on a departmental scale; the outcome is a picture of the haplotype

311 configuration within France (Figure 3). The resulting map shows finer-grained detail:

312 we can define at least four distinct groups, plus a more widespread component. In the

313 southwestern corner, the Basque samples clearly separate from the Gascon ones. In the

314 northwestern vertex, the Breton departments exhibit their very own haplotypic

315 signature, in agreement with the lower level of differentiation detected with the $F_{S T}$

316 analysis and the higher internal effective migration rate detected with EEMS. The same

317 was found for the northern and northeastern departments that display a clearly shared

318 haplotypic configuration. The southwestern department of Haute-Garonne (HG) and the

319 southeastern one of Bouches-du-Rhône $(\mathrm{BdR})$ present higher frequencies for some local

320 haplotypes that in other departments reached only lower frequencies. Otherwise, a more

321 generally spread French haplotypic background is found on the north-south axis.

322

\section{Sources of gene flow into France}

When we added external sources from the surrounding populations (yellow dots in Supplementary Figure 3) to describe allele-based genomic components with ADMIXTURE (Figure 4), the configuration observed pointed to a general homogeneous picture. The only exception was represented by the samples belonging to the Breton departments whose configuration was more alike to that in the Irish, Scottish, and English groups. Moving through the different $\mathrm{K}$ ancestral components, this behavior clearly characterizes the northwestern departments, separating them from the rest of the French groups since the very first K ancestral components (Figure 4). Thus, we formally tested for admixture events using the f3-statistics with the test groups being the different departments, and the external surrounding populations as sources. We only retained the negative $\mathrm{f} 3$ values for those departments represented at least by two individuals. Results are shown in Supplementary Table 2 were only significant Z-scores $<-3$ are reported, while results for those departments passing all the requested filters but with higher Z-score values are shown in Supplementary Table 3. Notably, in 9

339 departments, a combination of sources that was highly significant was Ireland-Southern 340 Italy. 
345 Based on the haplotype sharing with external sources it was possible to redefine the

346 French haplotype configuration. After merging the 395 French samples with the 1132

347 external ones (Dataset C), we first defined the external groups by silencing France when

348 rerunning ChromoPainter and fineSTRUCTURE. The result was represented by 35

349 different external groups (Supplementary Figure 8a). Secondly, focusing on our target,

350 we redefined the French internal clusters using the 35 external ones as "continents"

351 when running fineSTRUCTURE (Supplementary Figure 8b). The 13 different clusters

352 we found within France were then represented as separate maps (Figure 5); each map in

353 the figure is a heatmap showing the number of samples falling in the different

354 departments. Out of 13 groups, 10 satisfied the conditions of having at least 10

355 individuals and a major geographical area with a number of subjects corresponding to

356 more than $50 \%$ of the entire cluster. These conditions allowed us to name each cluster

357 based on the fact that a specific area was more represented than others in terms of

358 sample size. The exclusion of three clusters did not impact the analysis, since only

$3598.35 \%$ of the French samples were then not included as target in the following analyses

360 with GLOBETROTTER. As in the analysis described in the previous paragraph, even in

361 this case France appeared to be organized in few major areas of interest. As shown in

362 Figure 5, the Northwest presented two main groups (B1 and B2), the Southwest divided

363 in Basque (Bas) and Gascon (G1 and G2) groups, the Northern (CN) and Northeastern

364 (NE) areas, the Southeast (SE), and a central/southwestern part of France (CSW1 and

$365 \mathrm{SW}$ ). These ten main areas represented the targets for the GLOBETROTTER analysis

366 that we used to describe the ancestry profiles, the admixture events, and their dates.

\section{Ancestry profiles and dating admixture events}

370 The results from the application of the nnls algorithm are displayed in Figure 5; on both

371 sides of each target the ancestry profiles are represented as doughnut charts (on the left

372 the results from the NM analysis, on the right the ones for the $\mathrm{M}$ one). The different

373 colors represent proportions of haplotype sharing with specific sources (only

374 contributions above $2.5 \%$ are shown). In the NM analysis, it is possible to appreciate

375 how the haplotype sharing with other French sources (brown color) represents the

376 highest proportion for all the different targets. When masking the French component,

377 more refined patterns of contributions from external sources are detected. With the only 
378 exception of the southwestern targets (G1, G2, and Bas), the remaining ones show a

379 higher contribution from north Italy and Great Britain. Apart from these common

380 signal, it is possible to highlight contributions from those neighboring populations that

381 are more geographically close to specific areas within the French territory. The

382 southwestern targets (G1, G2, and Bas) received more from the Spanish side, the

383 northwestern targets (B1 and B2) share more with the external cluster source named

384 Irish_Scottish (with a proportion of $23.91 \%$ and $18.32 \%$ for the B1 and B2 targets

385 respectively), the northeastern target (NE) is more connected to the external cluster

386 source representing central and eastern European countries (receiving 17.64\% from the

387 source we named Central_Eastern_EU), as also from the NorthernEurope cluster

388 source (which contributes $7.35 \%$ and $5.78 \%$ to the NE and $\mathrm{CN}$ targets, respectively).

389 The southeastern target (SE) is mostly connected to the Italian sources and other

390 Mediterranean countries, and the central/southwestern target (CSW1) clearly received

391 more from both Spain and Italy.

393 As explained in Supplementary note 1, GLOBETROTTER provided evidence of

394 admixture for 8 out of 10 targets, and for 5 of them we could also describe the dates and

395 the sources of admixture as shown in Supplementary Figure 9. For three targets

396 GLOBETROTTER gave one-date as result, while for the remaining two one-date-

397 multiway was detected. In each case, only one date of admixture was detected; for the

398 one-date groups a single admixing couple of sources was described, while two couples

399 of sources were presented in the case of one-date-multiway. For a better interpretation

400 of the results, consider the caption from Supplementary Figure 9.

\section{Relations with ancient populations}

404 In the analysis with Dataset D, we first explored the position of France in the context of

405 other modern populations, and then we focused on the relation with a set of ancient

406 samples from different periods. In Supplementary Figure 10, panel A shows the PCA

407 with the modern samples; France (white circles) is located in a position that mirrors its

408 geographical situation, in between British, Irish, Mediterranean, central and eastern

409 European samples. In panel B, a set of ancient samples was projected into the modern

410 genetic space. In this second PCA, most of the French individuals are close to the

411 Steppe and the Late Neolithic Bronze Age (LNBA) European samples, with some 
412 subjects connecting with the Anatolian Neolithic and the Early Neolithic Eurpean

413 groups, and few others with the Europe Middle Neolithic and Chalcolithic

414 (Europe_MNChL) samples. Results from the ADMIXTURE analysis are reported for

415 the lowest cross-validation error detected $(K=4$ in Supplementary Figure 11). At this

416 level, four ancestral components are clearly visible: the hunter-gatherer (HG) ancestry

417 (principally represented by the Scandinavian HG, in pink), Neolithic (mostly Anatolian

418 and then European, in green), the Iran Neolithic (black), and Natufian (purple). Again,

419 the proportion of these components in France is intermediate between those in Southern

420 and Central European groups. It is especially the Natufian component that seems to act

421 as a discriminant factor, not only inside France where it is virtually absent with few

422 exceptions on the Mediterranean side, but mostly among the various modern groups.

423 Outgroup f3-statistics in the form of f3(Ancient, X; Mbuti) allowed us to quantify for

424 each X modern group the amount of shared drift with different ancient populations.

425 Figure 6 shows the outcome for these statistics, with a focus on the shared drift with the

426 three main European ancestral components: Western, Eastern, and Scandinavian hunter-

427 gatherers, European Neolithic farmers, and the European Bronze Age steppe

428 component. In most cases, French populations fit the expected pattern of distribution in

429 the wider panorama of the European area. However, the European Neolithic component

430 seems to be higher in the SW of France, while Brittany carries a proportion of HG

431 ancestry that is higher than elsewhere in France but closer to the values in the British

432 Isles.

Discussion

439 We have used both allele frequency and haplotype-based methods in order to describe

440 the internal structure of pre-20th century Metropolitan France. While the first yielded a

441 more homogeneous landscape, the latter unveiled patterns of local differentiation with

442 some connections with the surrounding European populations. Furthermore, we

443 explored patterns of genetic continuity with ancestral populations, contextualizing

444 France in the wider European panorama. In previous works about France, samples were 
445 differently arranged into the geographical space and no consensus had been reached on

446 what subdivision was more appropriate; apart from the peculiar military districts ${ }^{3}$,

447 historical provinces ${ }^{4,6}$ and old regions ${ }^{8}$ are the most used so far. Thus, our first goal

448 was to search for the best geographical level of genetic stratification before arranging

449 our samples on a map. After the French Revolution in 1790, in order to weaken the old

450 loyalties, the ancient provinces of France were subdivided into departments, whose

451 overall configuration has been mostly conserved so far ${ }^{30}$. Furthermore, in 1982, a

452 system of 22 regions was established by grouping different departments into wider areas

$4533^{31}$. However, in 2016, the number of the regions was reduced to 13, with the consequent

454 rearrangement of the departments ${ }^{32}$. Given this background, our AMOVA results

455 provide evidence that regions, as a new internal reorganization, are not a suitable model

456 for the genetic compartmentalization and point to the absence of any contribution to the

457 total genetic variation, possibly implying that regions are separating genetically similar

458 departments into different groups. On the other hand, departments, as result of a more

459 conserved internal geographical structure, represent the best minimal unit of genetic

460 stratification.

461

462 Dissecting the Hexagone

463 Principal component analysis on allele frequencies revealed the expected Basque

464 differentiation, adding Gascons in SW France as a population closely related to them,

465 while the rest of France appeared relatively homogeneous. However, EEMS results

466 pointed to the existence of other barriers to gene flow, particularly between NW France

467 (Brittany) and the rest, while other areas acted as corridors, in central France and along

468 the $\mathrm{N}$ and NE borders (Figure 2). It should not be excluded, though, that unsampled

469 regions caused some possible artifacts ${ }^{33}$. It was with fineSTRUCTURE that we could

470 really define a fine scale internal subdivision of France (Figure 3). A general

471 widespread French haplotypic background moving through the north-south axis was

472 detected; possibly the overall homogeneity found with the principal component analysis

473 can be linked to the fact that, on an allele frequency scale, such widespread pattern may

474 represent a confounding factor. Indeed, only the two Southwestern groups (Basques and

475 Gascons) were not reached by this common French haplotypic background. Particular

476 haplotype sharing patterns could also be observed along the north and northeast of

477 France, in the southeast, and among the northwestern departments. 
479 In order to understand whether these internal patterns of differentiation are due to recent

480 events or whether they reflect a more ancient history, we relied on different analyses

481 obtaining distinct information. On the one hand, we looked at the relation with modern

482 external populations, exploring both allele-frequency (ADMIXTURE and f3-statistics)

483 and haplotype-based methods (using GLOBETROTTER, we described the ancestry

484 profiles for 10 different French targets, defined by the haplotype sharing with external

485 sources, and provided a date of admixture events for 5 of them). On the other hand, we

486 looked for the continuity between modern France and ancestral populations from

487 different times.

488

489 France, carrefour of Europe

490 An ADMIXTURE plot (Figure 4), and a PCA with reference populations

491 (Supplementary Figure 10A) place most French populations as similar to their

492 geographic neighbours, namely the British Isles, Central Europe, Spain and Italy, in

493 accordance with the general observation in Europe of geographic distance as the main

494 predictor of genetic distance ${ }^{34,35}$. This may explain an apparently surprising outcome of

495 our work: 9 out of 22 distinct targets in $\mathrm{f} 3$ statistics we tested against different external

496 sources gave significant results with the lowest Z-scores detected for the same couple

497 represented by the South Italian and Irish sources. Z-scores lower than -3 indicate that

498 our test populations are admixed from sources not necessarily identical but related to the

499 sources we used in the analysis ${ }^{11}$. Interestingly, these results found support in the

500 outcome from the ancestry profiles we carried out with the Dataset C. The ancestry

501 profiles described in Figure 5 are informative of differential migratory patterns ${ }^{36}$ into

502 each of the ten French genetic targets. The ancestry profiles are a way to describe the

503 genome of each one of the ten French target as a mixture of the genomes from other

504 groups, without inferring any particular admixture event ${ }^{37}$. With this analysis, each

505 target is described as a composition of different proportions of haplotype sharing with

506 other sources, excluding the contribution of the group that we want to explain (no self-

507 copying allowed). Following the previous results from the f3-statistics, in the $\mathrm{M}$

508 analysis we found that 7 out of the 10 targets we tested were mostly described by high

509 proportions of haplotype sharing with both Italy and the British Isles. Furthermore, the

$510 \mathrm{NM}$ analysis highlighted the presence of a very strong shared French component,

511 possibly reflecting the result of a higher intermixing between individuals from the

512 different parts of modern France. 
514 An additional dimension to the central genetic position of France in Western Europe is

515 given by the comparison with a time transect of ancient samples. The ADMIXTURE

516 results for dataset D (Supplementary Figure 11), as well as the projected PCA

517 (Supplementary Figure 10B) place France again as intermediate between Southern and

518 Central Europe. However, this pattern is locally nuanced, as discussed below. Thus, it

519 appears that France has been operating as a crossroads for human migration in Western

520 Europe since, at least, the Early Neolithic.

\section{Basques and Gascons}

524 These groups clearly differentiated from the rest of France both with allele frequency

525 and with haplotype-based methods. It is interesting to notice that the presence of two

526 distinct groups in the Southwestern region stressed the outcome of the isolation the

527 Basque-speaking group experienced, splitting from their non-Basque-speaking

528 neighbors from the very same department (PA and PAB groups). This finding is in

529 agreement with their recognized distinct cultural entity ${ }^{38}$ and their genetic outlier

530 position in the European landscape ${ }^{39}$, as also with the lower internal levels of

531 differentiation we detected with the $F_{S T}$ analysis, and the low effective migration rates

532 evidenced by EEMS, resulting in a barrier to migration in the southwestern corner of

533 France.

534 The ancestry profile for French Basques (Figure 5) reflects an almost exclusive

535 component from Spanish Basques, with some minor contribution from two other source

536 clusters in the Iberian Peninsula. Quite often, Spanish populations are modelled as the

537 result of a Basque background plus external admixture ${ }^{40}$, so it is not surprising that

538 haplotypes found in Basques are also present in Spain. French and Spanish Basques, as

539 well as other populations in NE Iberia, share also an increase in shared drift with Early

540 Neolithic ancient samples (Figure 5D). The Basque singularity has often been explained

541 as due to the persistence of an ancient gene pool, as old as the Late Glacial ${ }^{41}$, or as the

542 Pre-Neolithic ${ }^{42}$, or as the Neolithic ${ }^{43}$ (as our results seems to suggest), but a recent

543 analysis of a large number of ancient Iberian samples ${ }^{44}$ points to a more recent

544 divergence, probably in the Iron Age, of the Basque population. 
546 Gascons have been shown to be intermediate between French Basques and other French

547 populations by PCA (Figure 1), and to carry a sizeable proportion of Basque ancestry

548 (Figure 5). This could be the result of the postulated contraction of the Basque-speaking

549 lands since the late Antiquity. Place names may indicate that Basque or languages

550 similar to it may have been spoken in Aquitaine (SW France) south of the Garonne river

55145 .

552

553

The Celtic connection

555 As shown by EEMS (Figure 2), a barrier to gene flow delineates the northwestern

556 corner of France, indicating the presence of another distinct group represented by the

557 Breton departments. This group was firstly detected, on a coarser scale, with the

558 removal of the Southwestern samples (Basques and Gascons) from the first PCA, and

559 its outstanding position is in agreement with different studies on both uniparental and

560 autosomal markers ${ }^{6-9}$. However, based on the fineSTRUCTURE results, in our work

561 we detected a stronger evidence of differentiation based on haplotypic data.

562 ADMIXTURE showed a connection to the Irish samples (Figure 4), which is also

563 indicated by the ancestry profiles of the B1 and B2 targets, which showed higher

564 proportions for the Irish_Scottish cluster source (Figure 5). The GLOBETROTTER

565 analysis for determining the admixture dates pointed to some interesting results

566 (Supplementary Figure 9). B2, the largest Breton target, gave signals of admixture

567 around $700 \mathrm{CE}$, in the time frame of the British Celtic migrations (from Cornwall and

568 south-west Britain) into Gaulish Armorica (then renamed Brittany) from the 3rd to 9th

569 centuries $\mathrm{CE}$, with a higher flow between the 5 th and the 6 th centuries $\mathrm{CE}^{46}$.This

570 completely agrees with previous findings ${ }^{7-9}$. Historical migrations from Ireland to

571 Brittany are well recorded since the 4 th century $\mathrm{CE}^{47}$, as well as the emigration of Irish

572 people during the War of Ireland (1641-1651) into the present day departments of

573 Finistère (FI) and Côte d'Armor (CdA), within which a higher integration of the Irish

574 immigrants is proved by records of marriage, birth and death certificates ${ }^{7}$. Furthermore,

575 a Celtic root for the Breton language links the Breton departments to the Insular Celtic

576 languages from the British Isles ${ }^{48}$.

577 Still, the connection may be more ancient. In Figure 6, we explore the three main

578 European ancestral components ${ }^{49}$ : the pre-Neolithic hunter-gatherers, the European

579 Neolithic farmers, and the European Bronze Age steppe. Observing the shared drift with 
580 the three hunter-gatherer groups (panels A, B, and C), it is possible to notice how the

581 northwestern departments are mirroring the values shown by the British Isles, the

582 Central-Eastern countries, and Northern Europe. Brittany is thus showing a signal of

583 continuity with the British Isles which could be ascribed to a period older than the later

584 Celtic migration. Always Brittany is acting as an outlier in the case of the shared drift

585 with the Steppe Early and Middle Bronze Age group. In Figure 6 (panel E) it is possible

586 to see how Brittany breaks the northeast-to-southwest decreasing gradient of shared

587 drift. Even in this context, Brittany shows a continuity with the British Isles. Actually,

588 this is consistent with the archaeological records and the development of a late

589 Megalithic culture that characterized Ireland, Britain and Brittany in a period when

590 other parts of Europe were experiencing the advent of metallurgy ${ }^{50}$.

591

592

Borderlands

593 The northeastern rim of France, and the Mediterranean southeastern region represent

594 areas in the perimeter of the Hexagone that may have received particular genetic

595 influences. In the ancestry profiles (Figure 5), the NE and SE targets exhibit the most

596 complex genetic make-ups, with a diverse array of sources. The Central_Eastern_EU

597 cluster source is mostly represented in the NE target, which includes the departments of

598 Bas-Rhin and Moselle; this area recalls the long history of the Alsace-Lorraine territory:

599 a fuzzy border between France and Germany for a long time, and only recently

600 retroceded to France in $1945^{51}$.

601

602

603 The SE target (most abundant in the Bouches-du-Rhône department) copied from

604 several Mediterranean sources (thus representing the target with more complexity). This

605 area has been a corridor and a landing place for different Mediterranean peoples, since

606600 BCE when Greeks established a colony on the Mediterranean coastline of France in

607 the city of Massalia (present-day Marseille) ${ }^{52}$. However, this Mediterranean connection

608 may be older, since the late Epipaelolithic Natufian component (Supplementary Figure

609 11), which is found almost exclusively in the Mediterranean populations, is found in

610 France in the highest frequency in the Bouches-du-Rhône department.

\section{Conclusions}


615 In conclusion, according to our results, France is a genetic intermediate between

616 Central, Eastern, and Northern Europe, with some influences from the Mediterranean

617 countries on the southeastern coast. Analyses with both modern and ancient groups

618 pointed to a clear separation of the southwestern groups (Basques and Gascons) and of

619 Brittany from the rest of the French areas. The application of haplotype-based methods

620 allowed us to look beyond the more homogeneous French haplotypic background,

621 discovering connections with the neighbouring populations (e.g., French northeastern

622 departments with central and eastern Europe), while analyses with ancestral populations

623 strengthened the historical connection between Brittany and the British Isles.

\section{DATA AVAILABILITY}

626 The genotypes of the samples typed for this manuscript can be downloaded from

627 https://figshare.com/articles/France_Dataset/10008689

628 and https://figshare.com/articles/Naples_Dataset/10008731

629

\section{ACKNOWLEDGMENTS}

631 We thank Inés Quintela and the National Genotyping Center (CEGEN - USC) for their assistance in genotyping the samples. Of course, this work would not have been possible without the kind collaboration of all the sample donors. Funding was provided

634 by the Agencia Estatal de Investigación and Fondo Europeo de Desarollo Regional 635 (FEDER) (grant CGL2016-75389-P), Agència de Gestió d'Ajuts Universitaris i de la 636 Recerca (Generalitat de Catalunya) grant 2014 SGR 866, and "Unidad de Excelencia 637 María de Maeztu”, funded by the MINECO (ref: MDM-2014-0370). SAB was 638 supported by the Agencia Estatal de Investigación FPI grant BES-2014-069224.

AUTHOR CONTRIBUTIONS

$641 \mathrm{SAB}$ and FC designed the study; SAB carried out the analyses and interpretations,

642 which were discussed with FC and DC; ERL and DC provided samples and unpublished 643 genotypes. SAB wrote a first draft of the manuscript, with contributions from FC and

644 DC. All authors read and approved the last version of the manuscript. 


\section{References}

648

649 1. Haine, W. S. The history of France. (Greenwood Press, 2000).

650 2. MacMaster, N. Colonial Migrants and Racism Algerians in France, 1900-62.

651 (Palgrave Macmillan, 1997).

$652 \quad 3 . \quad$ Kherumian, R., Moullec, J. \& Nguyen, V. C. Groupes sanguins érythrocytaires A , A , BO, MN, Rh (CcDE) et sériques, $\mathrm{Hp}, \mathrm{Tf}, \mathrm{Gm}$ dans quatre régions militaires françaises. Bull. Mem. Soc. Anthropol. Paris 1, 377-384 (1967).

4. Cambon-Thomsen, A. \& Ohayon, E. Practical Application of Population Genetics: The Genetic Survey "Provinces Françaises". in Advances in Forensic Haemogenetics. Advances in Forensic Haemogenetics, vol 2. (ed. Mayr, W. R.) 535-553 (1988).

5. Cavalli-Sforza, L. L., Menozzi, P. \& Piazza, A. The History and Geography of Human Genes. (Princeton University Press, 1994).

6. Richard, C. et al. An mtDNA perspective of French genetic variation. Ann. Hum.

Biol. 34, 68-79 (2007).

664

7. Dubut, V. et al. mtDNA polymorphisms in five French groups: importance of regional sampling. Eur. J. Hum. Genet. 12, 293-300 (2004).

666

8. Ramos-Luis, E. et al. Y-chromosomal DNA analysis in French male lineages.

Forensic Sci. Int. Genet. 9, 162-168 (2014).

667 9. Karakachoff, M. et al. Fine-scale human genetic structure in Western France.

668 Eur. J. Hum. Genet. 23, 831-836 (2015).

669 10. Patterson, N. et al. Ancient Admixture in Human History. Genetics 192, 10651093 (2012).

11. Lazaridis, I. et al. Genomic insights into the origin of farming in the ancient Near East. Nature 536, 419-424 (2016).

12. Martínez-Cruz, B. et al. Evidence of pre-Roman tribal genetic structure in Basques from uniparentally inherited markers. Mol. Biol. Evol. 29, 2211-22 (2012).

13. Biagini, S. A. et al. People from Ibiza: an unexpected isolate in the Western Mediterranean. Eur. J. Hum. Genet. 27, 941-951 (2019).

678 14. Purcell, S. et al. PLINK: a tool set for whole-genome association and populationbased linkage analyses. Am. J. Hum. Genet. 81, 559-75 (2007). 
680 15. Patterson, N., Price, A. L. \& Reich, D. Population structure and eigenanalysis.

681

682

683

684

685

686

687

688

689

690

691

692

693

694

695

696

697

698

699

700

701

702

703

704

705

706

707

708

709

710

711

712

713 PLoS Genet. 2, 2074-2093 (2006).

16. RStudio Team. RStudio Team (2015). RStudio: Integrated Development for R. (2015).

17. R Core Team. R: A Language and Environment for Statistical Computing. R Foundation for Statistical Computing, Vienna. (2018).

18. Dray, S. \& Dufour, A.-B. The ade4 Package: Implementing the Duality Diagram for Ecologists. J. Stat. Softw. 22, (2007).

19. Wickham, H. ggplot2. (Springer New York, 2009). doi:10.1007/978-0-38798141-3.

20. Wickham, H. Reshaping Data with the reshape Package. J. Stat. Softw. 21, (2007).

21. Kamvar, Z. N., Brooks, J. C. \& Grünwald, N. J. Novel R tools for analysis of genome-wide population genetic data with emphasis on clonality. Front. Genet. 6, (2015).

22. Kamvar, Z. N., Tabima, J. F. \& Grünwald, N. J. Poppr : an R package for genetic analysis of populations with clonal, partially clonal, and/or sexual reproduction. PeerJ 2, e281 (2014).

23. Alexander, D. H. \& Novembre, J. Fast Model-Based Estimation of Ancestry in Unrelated Individuals. Genome Res. 19, 1655-1664 (2009).

24. Behr, A. A., Liu, K. Z., Liu-Fang, G., Nakka, P. \& Ramachandran, S. pong: fast analysis and visualization of latent clusters in population genetic data. Bioinformatics 32, 2817-2823 (2016).

25. Petkova, D., Novembre, J. \& Stephens, M. Visualizing spatial population structure with estimated effective migration surfaces. Nat. Genet. 48, 94-100 (2016).

26. O'Connell, J. et al. A General Approach for Haplotype Phasing across the Full Spectrum of Relatedness. PLoS Genet. 10, e1004234 (2014).

27. Delaneau, O. et al. Integrating sequence and array data to create an improved 1000 Genomes Project haplotype reference panel. Nat. Commun. 5, 1-9 (2014).

28. Lawson, D. J., Hellenthal, G., Myers, S. \& Falush, D. Inference of Population Structure using Dense Haplotype Data. PLoS Genet. 8, e1002453 (2012).

29. Hellenthal, G. et al. A genetic atlas of human admixture history. Science (80-. ). 343, 747-751 (2014). 
714 30. Forstenzer, T. R. French Provincial Police and the Fall of the Second Republic:

715 Social Fear and Counterrevolution. (Princeton University Press, 2016).

716 31. Sowerwine, C. France since 1870: Culture, Society and the Making of the

717 Republic. (Palgrave Macmillan, 2009).

718

32. OECD. OECD Multi-level Governance Studies Multi-level Governance Reforms Overview of OECD Country Experiences. (2017).

33. House, G. L. \& Hahn, M. W. Evaluating methods to visualize patterns of genetic differentiation on a landscape. Mol. Ecol. Resour. 18, 448-460 (2018).

34. Novembre, J. et al. Genes mirror geography within Europe. Nature 456, 98-101 (2008).

35. Lao, O. et al. Correlation between Genetic and Geographic Structure in Europe. Curr. Biol. 18, 1241-1248 (2008).

36. Leslie, S. et al. The fine-scale genetic structure of the British population. Nature 519, 309-314 (2015).

37. Hellenthal, G. Instruction Manual for " GLOBETROTTER : a program for identifying, dating and describing admixture events in population data " for GLOBETROTTER. 1-24 (2015).

38. Calafell, F. \& Bertranpetit, J. Principal component analysis of gene frequencies and the origin of Basques. Am. J. Phys. Anthropol. 93, 201-215 (1994).

39. Rodríguez-Ezpeleta, N. et al. High-density SNP genotyping detects homogeneity of Spanish and French Basques, and confirms their genomic distinctiveness from other European populations. Hum. Genet. 128, 113-117 (2010).

40. Bycroft, C. et al. Patterns of genetic differentiation and the footprints of historical migrations in the Iberian Peninsula. Nat. Commun. 10, 551 (2019).

41. Pereira, L. et al. High-resolution mtDNA evidence for the late-glacial resettlement of Europe from an Iberian refugium. Genome Res. 15, 19-24 (2005).

42. Calafell, F. \& Bertranpetit, J. A simulation of the genetic history of the Iberian Peninsula. Curr. Anthropol. 34, 735-745 (1993).

43. Günther, T. et al. Ancient genomes link early farmers from Atapuerca in Spain to modern-day Basques. Proc. Natl. Acad. Sci. U. S. A. 112, 11917-22 (2015).

44. Olalde, I. et al. The genomic history of the Iberian Peninsula over the past 8000 years. Science 363, 1230-1234 (2019).

746 45. Zuazo, K. El euskera y sus dialectos. (Alberdania, 2010).

747 46. Koch, J. Breton Migrations. in Celtic Culture : A Historical Encyclopedia 275- 
277 (ABC-CLIO, 2005).

47. Monnier, J. Chapitre 6 : L'immigration bretonne en Armorique. in Toute

l'histoire de Bretagne (eds. Monnier, J. \& Cassard, J.) 97-106 (Skol Vreizh,

751 1997).

752

48. Forster, P. \& Toth, A. Toward a phylogenetic chronology of ancient Gaulish, Celtic, and Indo-European. Proc. Natl. Acad. Sci. 100, 9079-9084 (2003).

49. Lazaridis, I. et al. Ancient human genomes suggest three ancestral populations for present-day Europeans. Nature 513, 409-413 (2014).

50. Arias, P. The Origins of the Neolithic Along the Atlantic Coast of Continental Europe: A Survey. J. World Prehistory 13, 403-464 (1999).

51. Blumenthal, D. Alsace-Lorraine: A Study of the Relations of the Two Provinces to France and to Germany, and a Presentation of the Just Claims of Their People (Classic Reprint). (Forgotten Books, 2012).

52. Fine, J. V. A. The Ancient Greeks: A Critical History. (Belknap Press: An Imprint of Harvard University Press, 1985).

53. 1000 Genomes Project Consortium et al. A global reference for human genetic variation. Nature 526, 68-74 (2015).

54. Busby, G. B. J. et al. The Role of Recent Admixture in Forming the 


\section{$770 \quad$ Main Figures and Tables}

771

\begin{tabular}{llccc}
\multicolumn{1}{c}{ Groupings } & \% Total variance & $\Phi_{\text {-statistics }}$ & $p$ \\
\cline { 2 - 5 } A & Variations Between Areas & 0.02 & $\Phi_{\text {ST }}=0.0026$ & 0.4605 \\
Variations Between Departments Within Areas & 0.23 & $\Phi_{\text {ST }}=0.0023$ & 0.0009 \\
Variations Within Departments & 99.73 & $\Phi_{\text {ST }}=0.00027$ & 0.0009 \\
\hline & Variations Between Regions & -0.054 & $\Phi_{\text {ST }}=-0.0005$ & 0.7362 \\
B Variations Between Departments Within Regions & 0.3 & $\Phi_{\text {ST }}=0.003$ & 0.0009 \\
Variations Within Departments & 99.74 & $\Phi_{\text {ST }}=0.0025$ & 0.0009 \\
\hline
\end{tabular}

774 Table 1. Hierarchical analysis of molecular variance (AMOVA). Results for percentage of total variance,

$775 \Phi$-statistics, and p-values are reported for the three distinct analyses. A) proportion of genetic variation

776 partitioned among geographic areas, among departments within geographic areas, and within

777 departments; B) proportion of genetic variation partitioned among regions, among departments within

778 regions, and within departments; $\mathbf{C}$ ) proportion of genetic variation partitioned among departments and

779 within departments 


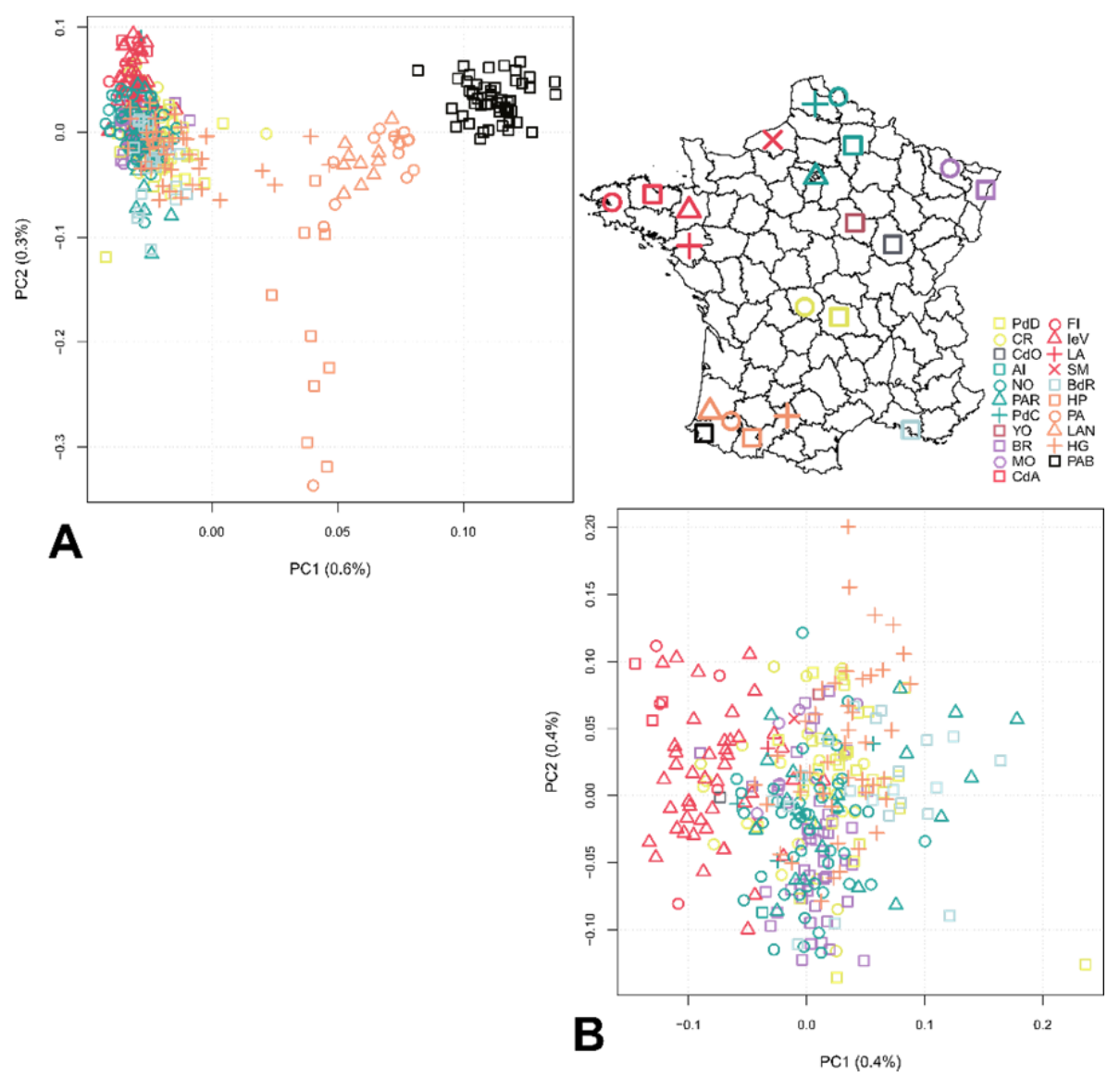

Figure 1. Principal Component Analysis of French samples (dataset A) with A) Basque and Gascon samples, and B) without them. Colors correspond to distinct geographic areas, while different symbols with the same color represent distinct departments in each area (See map distribution). However, Basques are colored differently than the nonBasque-speaking samples from that same area, but symbols recall the departments they share with the non-Basque-speaking groups. 


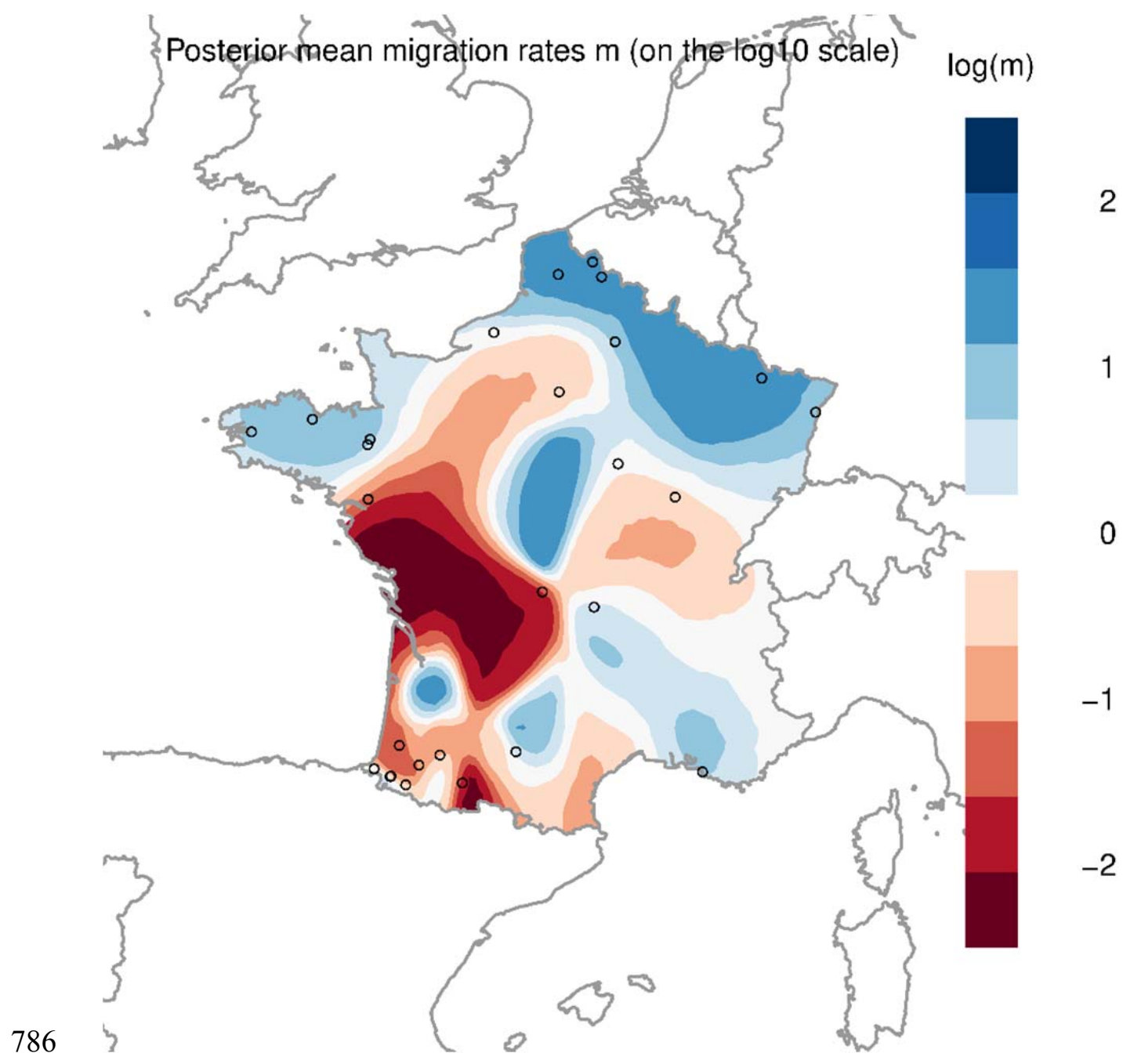

787 Figure 2. EEMS plot based on 395 French samples (Dataset A). Different shades of the same color

788 represent differential levels of high (blue) or low (red) effective migration rates. The zero value indicates

789 the average effective migration rate. Geographical locations for the different departments are averages of

790 the coordinates among samples. 
Figure 3. Pie charts showing the spatial distribution of haplotypes inferred by the fineSTRUCTURE tree. Each pie chart is a department, while colors correspond to the

clusters described in the tree above the map. See Figure 1 for department names. Asterisks indicate departments with only one sample. 
bioRxiv preprint doi: https://doi.org/10.1101/718098; this version posted October 28, 2019. The copyright holder for this preprint (which was not certified by peer review) is the author/funder, who has granted bioRxiv a license to display the preprint in perpetuity. It is made available under aCC-BY-NC-ND 4.0 International license.

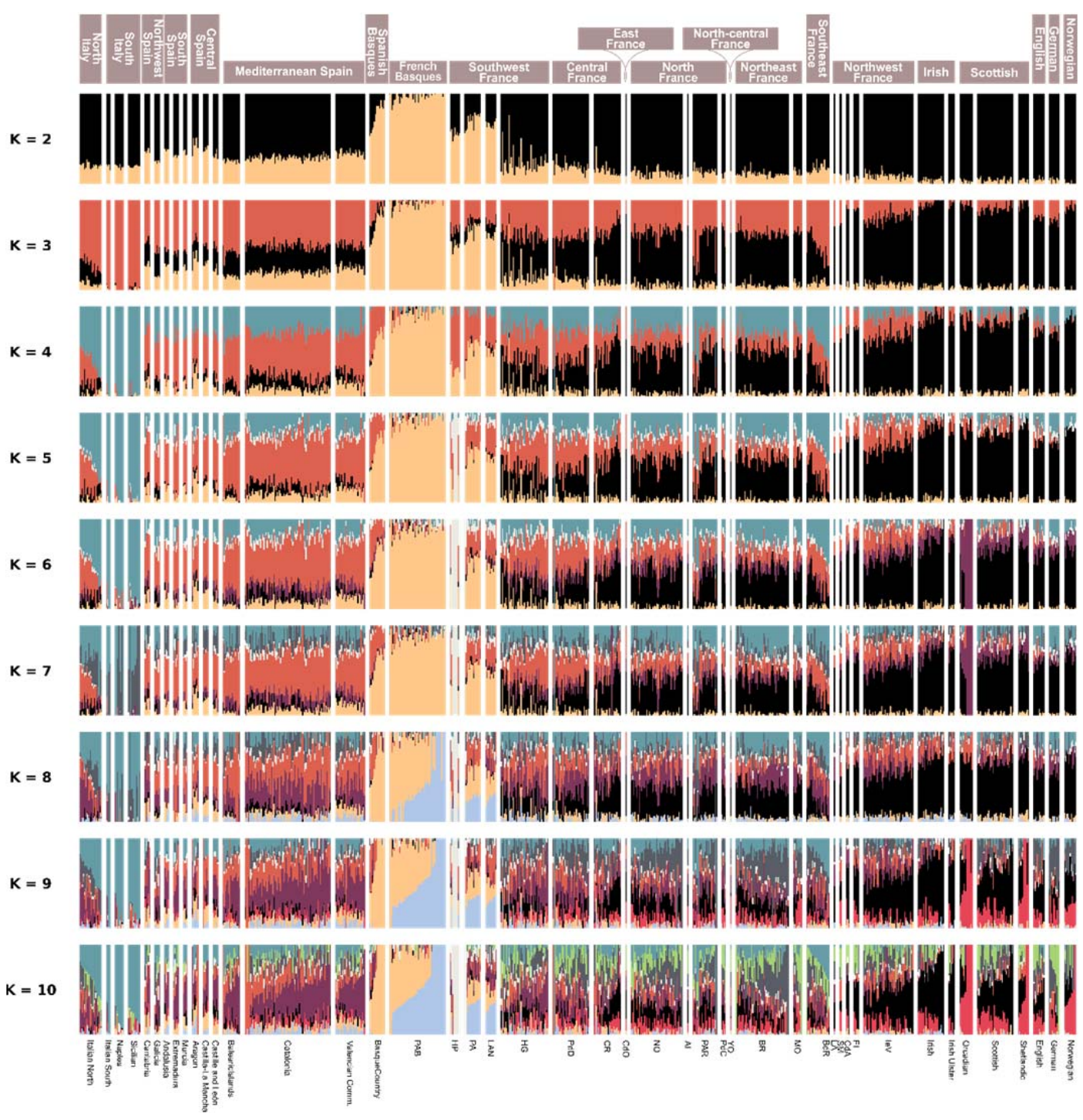

Figure 4. ADMIXTURE results from $\mathrm{K}=2$ to $\mathrm{K}=10$ for the 395 French samples (Dataset A) divided in cross-validation error was found with $\mathrm{K}=2$. 

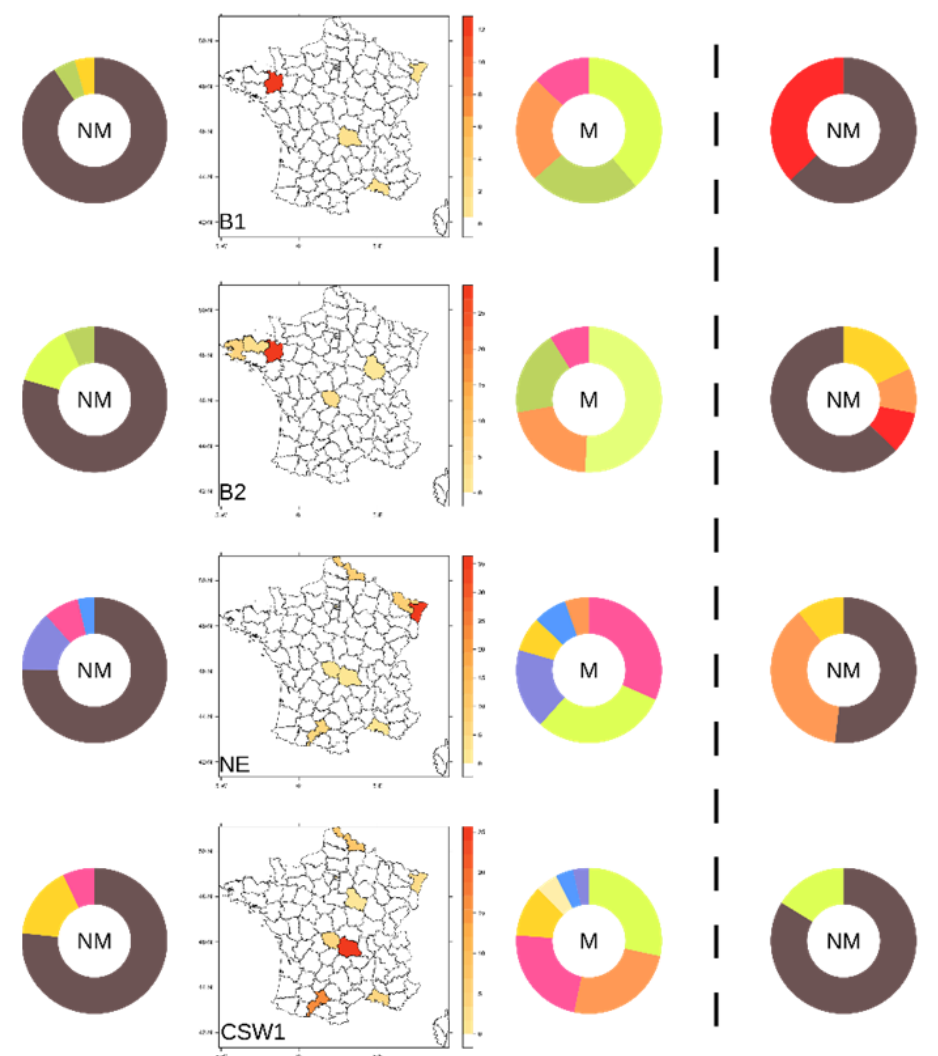

I
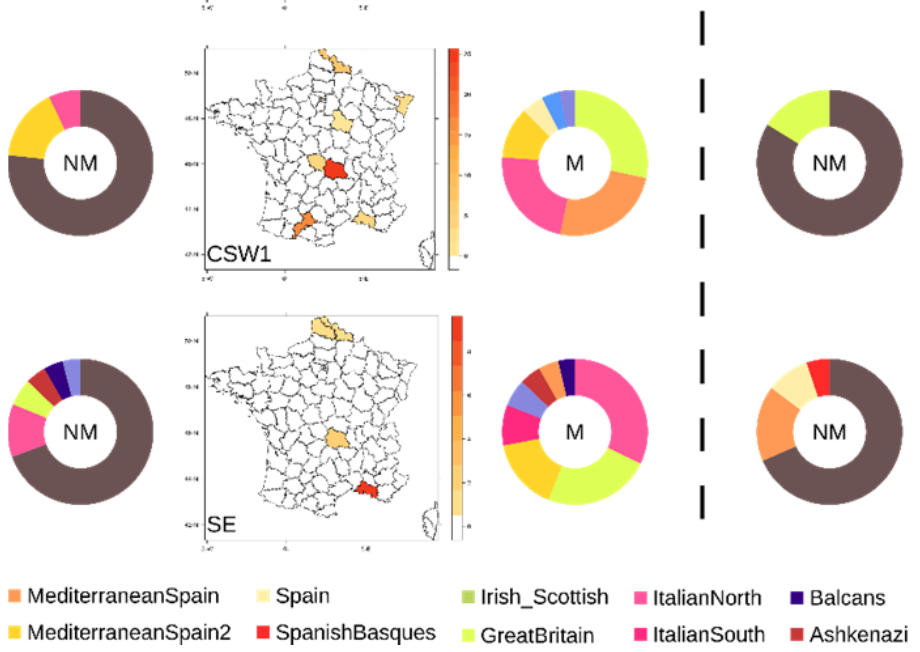

$=$ Irish_Scottish $=$ ItalianNorth $\quad$ Balcans
GreatBritain $\quad$ ItalianSouth $\quad$ Ashkenazi
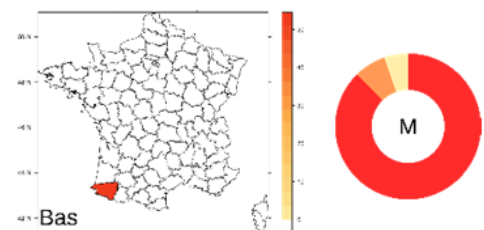

Bas
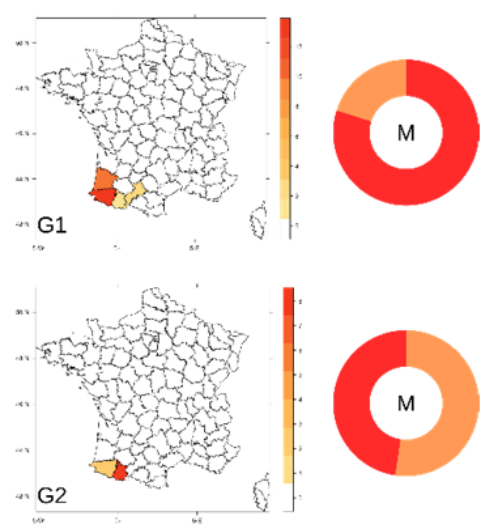

800

Figure 5. Ancestry profiles for 10 French targets. Each map is a target defining a specific major area of the French territory. On the left of each map, the donut chart is representing the ancestry profile for the not masked analysis (NM); on the right the same analysis has been masked (M). The different colors represent proportions of haplotype sharing with a specific source (only contributions above the $2.5 \%$ are shown); sources are defined in supplementary Figure 8. In the NM analysis, the brown color refers to contributions coming from other French groups (cumulative value). Target names stand for: B1 and B2, Brittany; NE, NorthEast; CSW1, Central-SouthWest; SE, SouthWest; Bas, Basques; G1 and G2, Gascons; CN, Central-North; SW, SouthWest. 


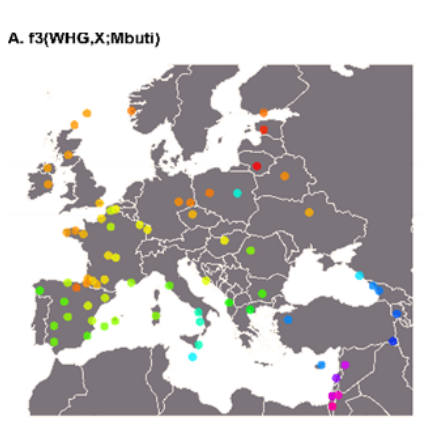

D. f3(Europe_EN,X;Mbuti)

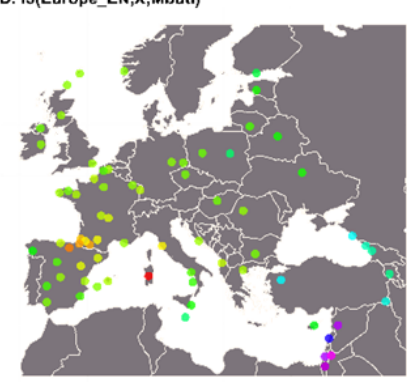

B. 13(EHG,X;Mbuti)

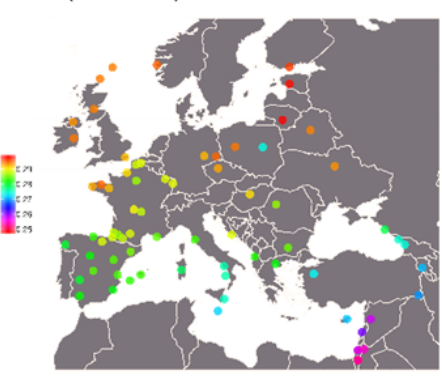

E. f3(Steppe_EMBA,X;Mbuti)

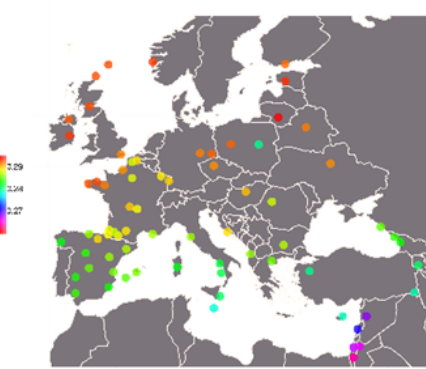

C. f3(SHG,X;Mbuti)

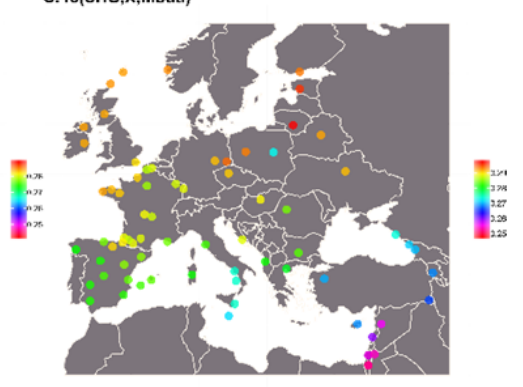

$m_{i x}^{m}$

Figure 6. Maps showing the distribution of the shared drift between different ancestral populations and the modern ones (X in the f3 statistics). Panels: A) f3(Western Hunter Gatherers,X;Mbuti), B) f3(Eastern Hunter Gatherers,X;Mbuti), C) f3(Scandinavian Hunter Gatherers,X;Mbuti), D) f3(Europe_Early Neolithic ,X;Mbuti), E) f3(Steppe Early Middle Bronze Age,X;Mbuti). In France, departments with less than two individuals are not shown. 


\section{Supplementary Data}

828

829

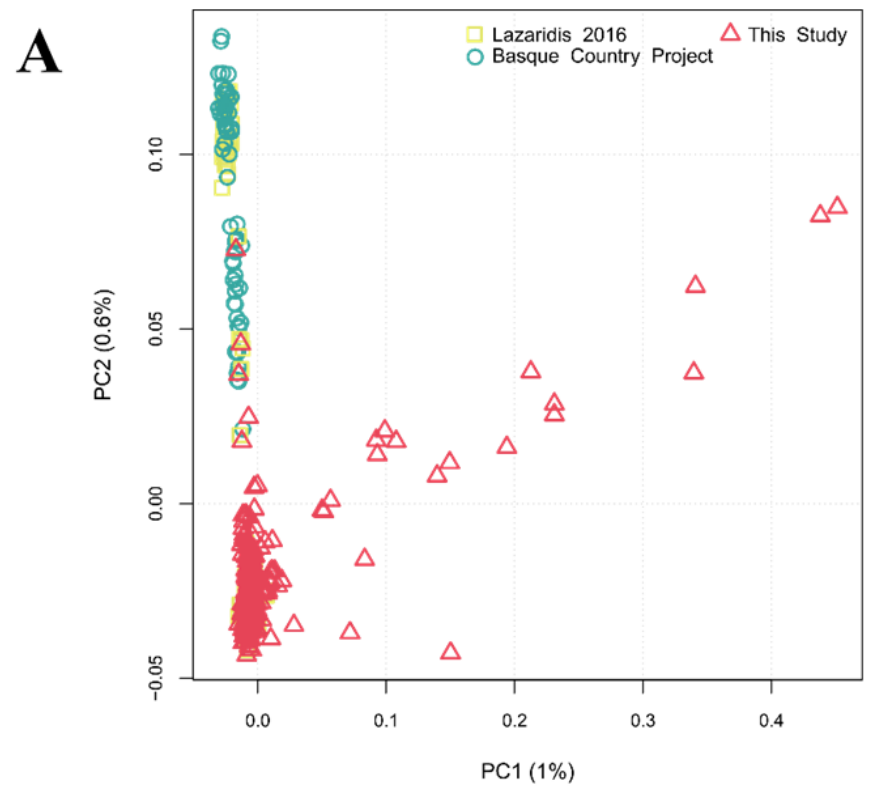

B

831 Supplementary Figure 1. PCA with 415 French samples highlighted the presence of outliers clearly

832 skewing the global distribution of the samples (A). We assessed the origin of those samples using

833 ChromoPainter and fineSTRUCTURE in the context of external references from three worldwide

834 populations (CEU, YRI, CHB) from the 1000 genomes project ${ }^{53}$ and North African samples from

835 published data ${ }^{11}$. Four clusters were defined (B), assigning the majority of our samples (395) to the

836 European cluster. The remaining 20 were outliers mainly belonging to the North African cluster (16

837 samples), 2 samples each were instead assigned to the Asian and the Sub-Saharan African clusters.

838

839

840 


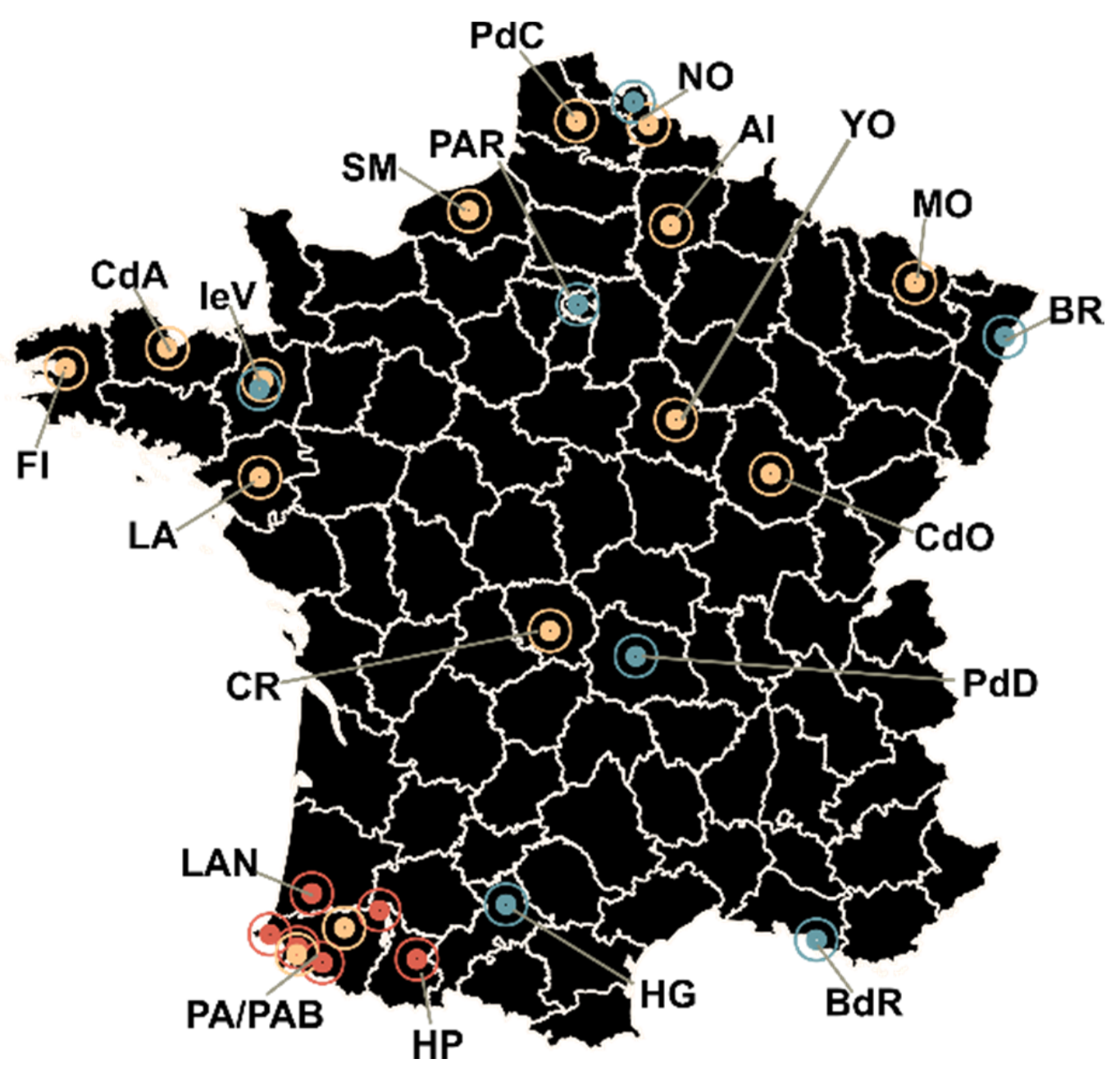

Supplementary Figure 2. Map showing sample distribution among the different departments.

Geographical coordinates are averages among samples. Different colors define the three datasets used in this work (blue dots correspond to the 256 samples genotyped for this work; yellow dots correspond to the 79 samples from Lazaridis et al., 2016; red dots correspond to the 60 samples from unpublished data). Sample size and acronyms for the departments are: PdD, Puy-de-Dôme (33); CR, Creuse (25); CdO, Côte-d'Or (1); AI, Aisne (1); NO, Nord (47); PdC, Pas-de-Calais (4); PAR, Paris (22); YO, Yonne (1); MO, Moselle (8); BR, Bas-Rhin (48); IeV, Ille-et-Vilaine (45); CdA, Côtes-d'Armor (3); FI, Finistère (5); SM, Seine-Maritime (2); LA, Loire-Atlantique (1); BdR, Bouches-du-Rhône (21); LAN, Landes (10); HG, Haute-Garonne (43); PA, Pyrénées-Atlantiques (15); PAB, Pyrénées-Atlantiques Basque (31); HP, 


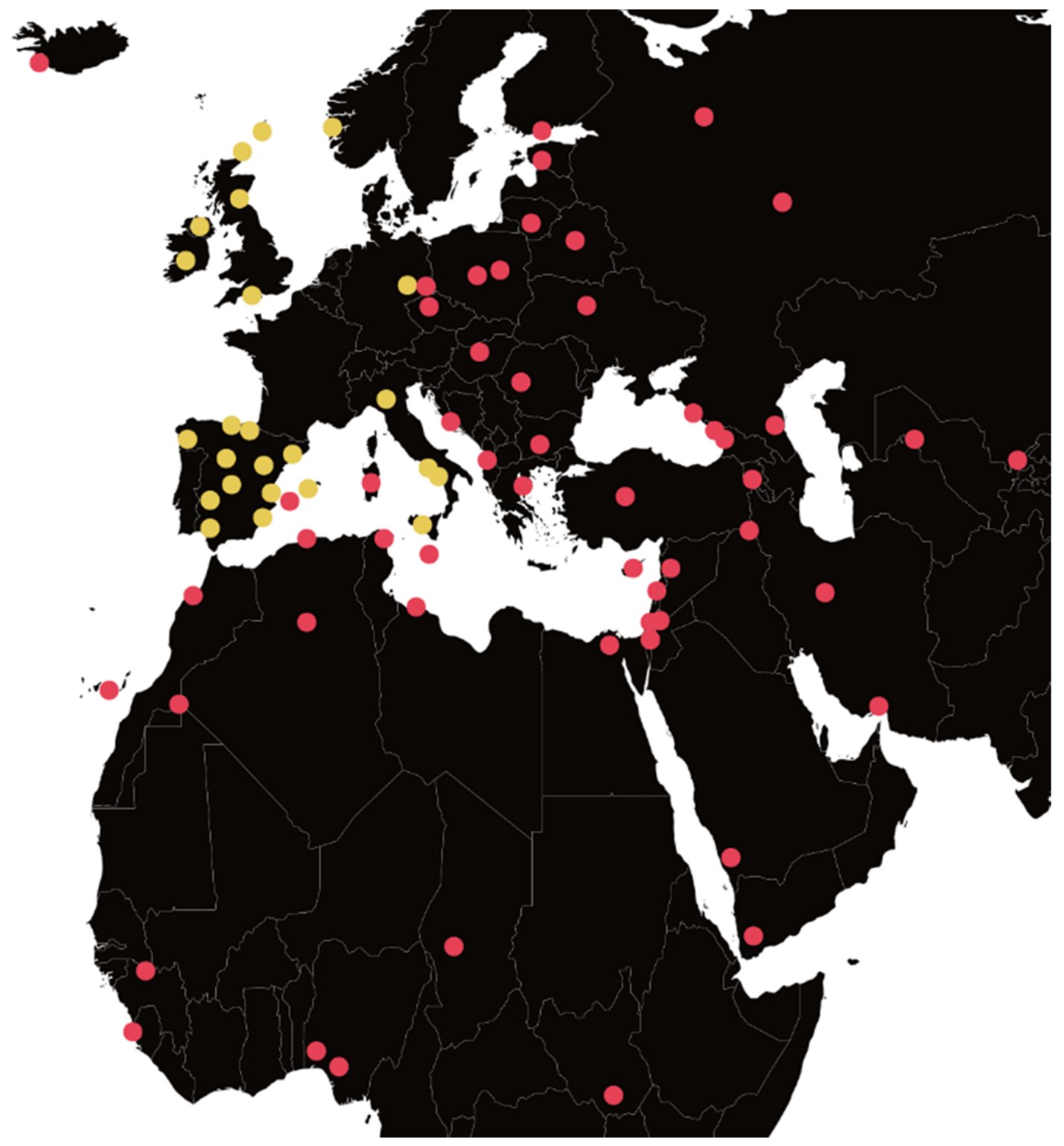

858 Supplementary Figure 3. External group distribution. Average geolocation points for the 79 external

859 populations are displayed. Yellow dots refer to the 333 samples included in the allele frequency analyses.

860 Yellow and red points together represent the 1132 samples used in the haplotype-based analyses 

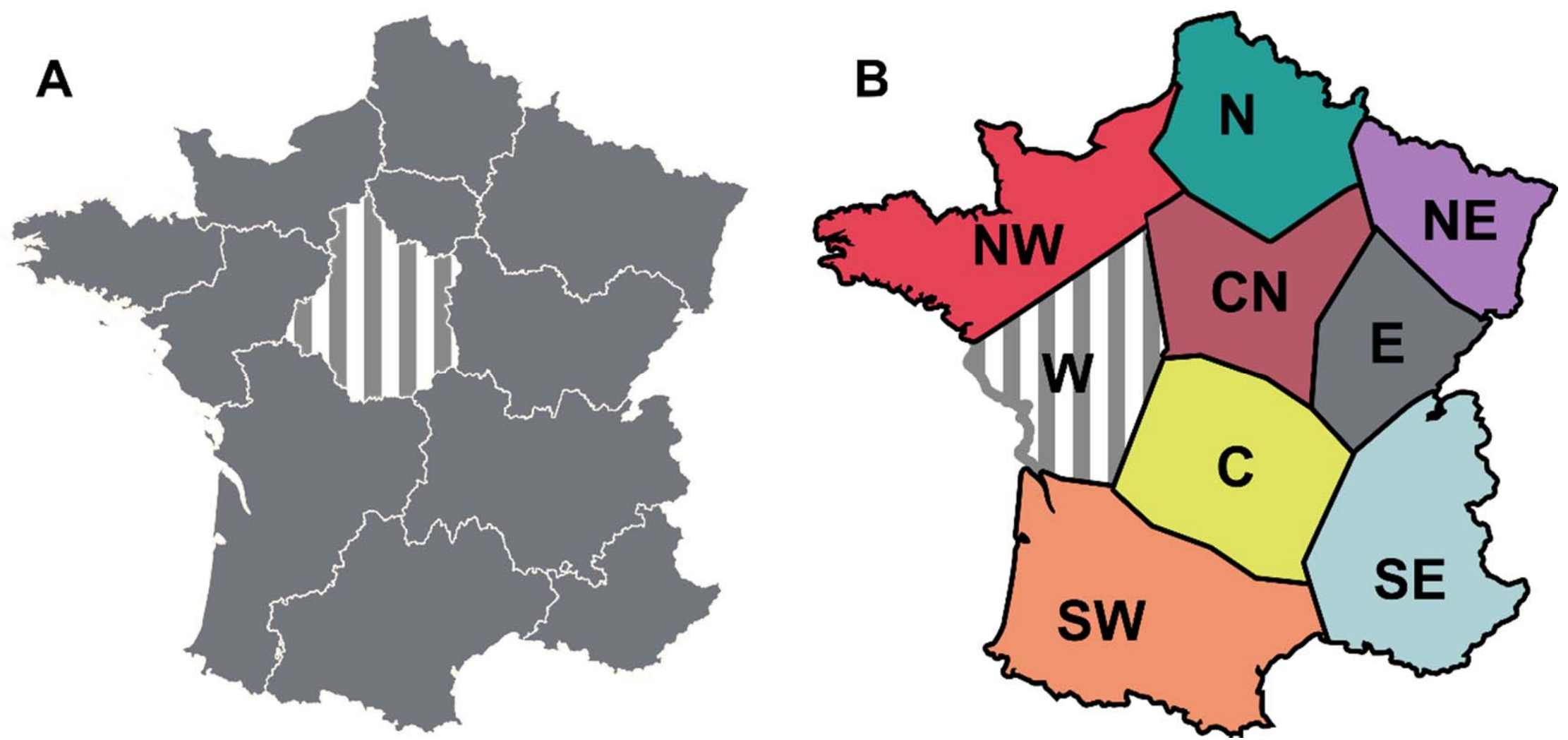

Supplementary Figure 4. Higher hierarchical levels used in the AMOVA analysis for A) Regions and B) Areas. Grey vertical lines highlight unsampled zones. Acronyms for the Areas are: NW, Northwest; N, North; NE, Northeast; W, West; CN, Central North; E, East; C, Center; SW, Southwest; SE, Southeast. 


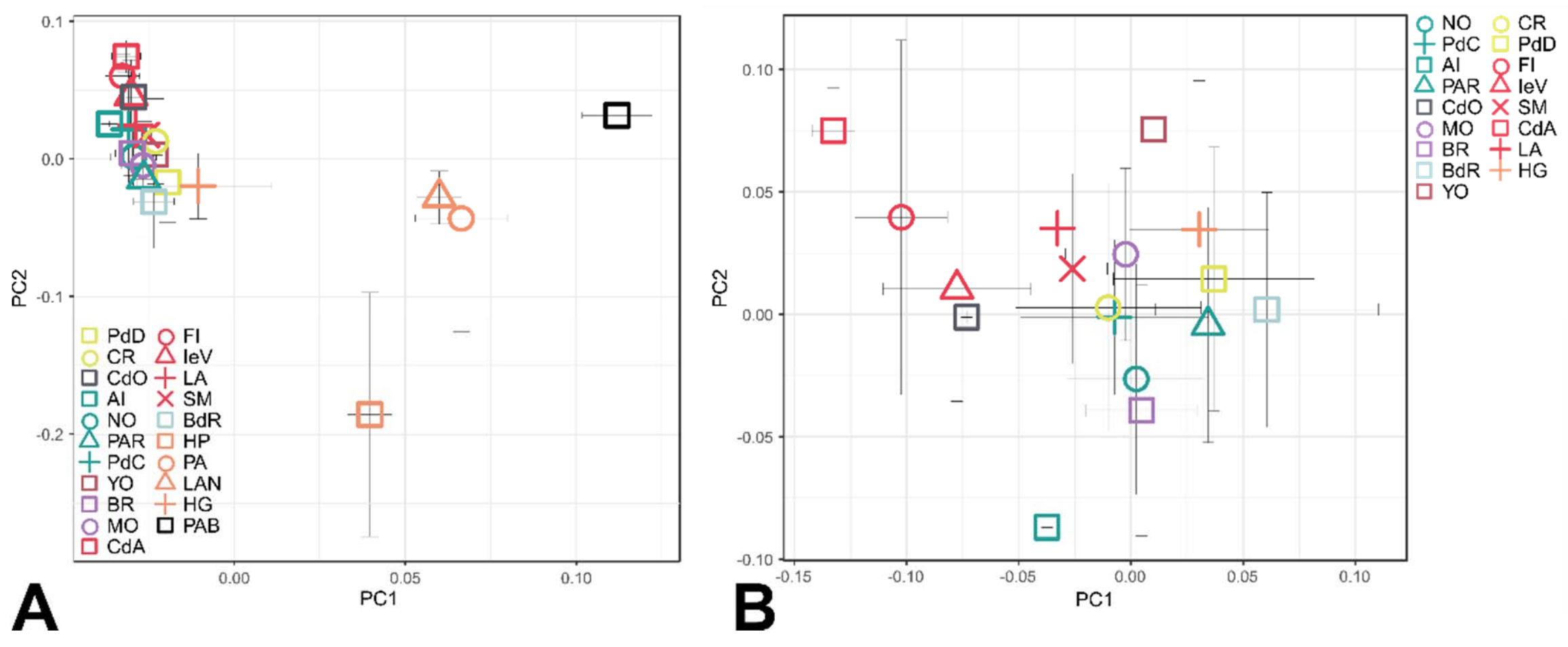

Supplementary Figure 5. Averaged Principal Component Analysis with A) Basque and Gascon samples, and B) without them. Color and symbol codes are the same as in main Figure 2. For each group, each averaged eigenvalue is represented along with standard deviation bars for the two PCs. 

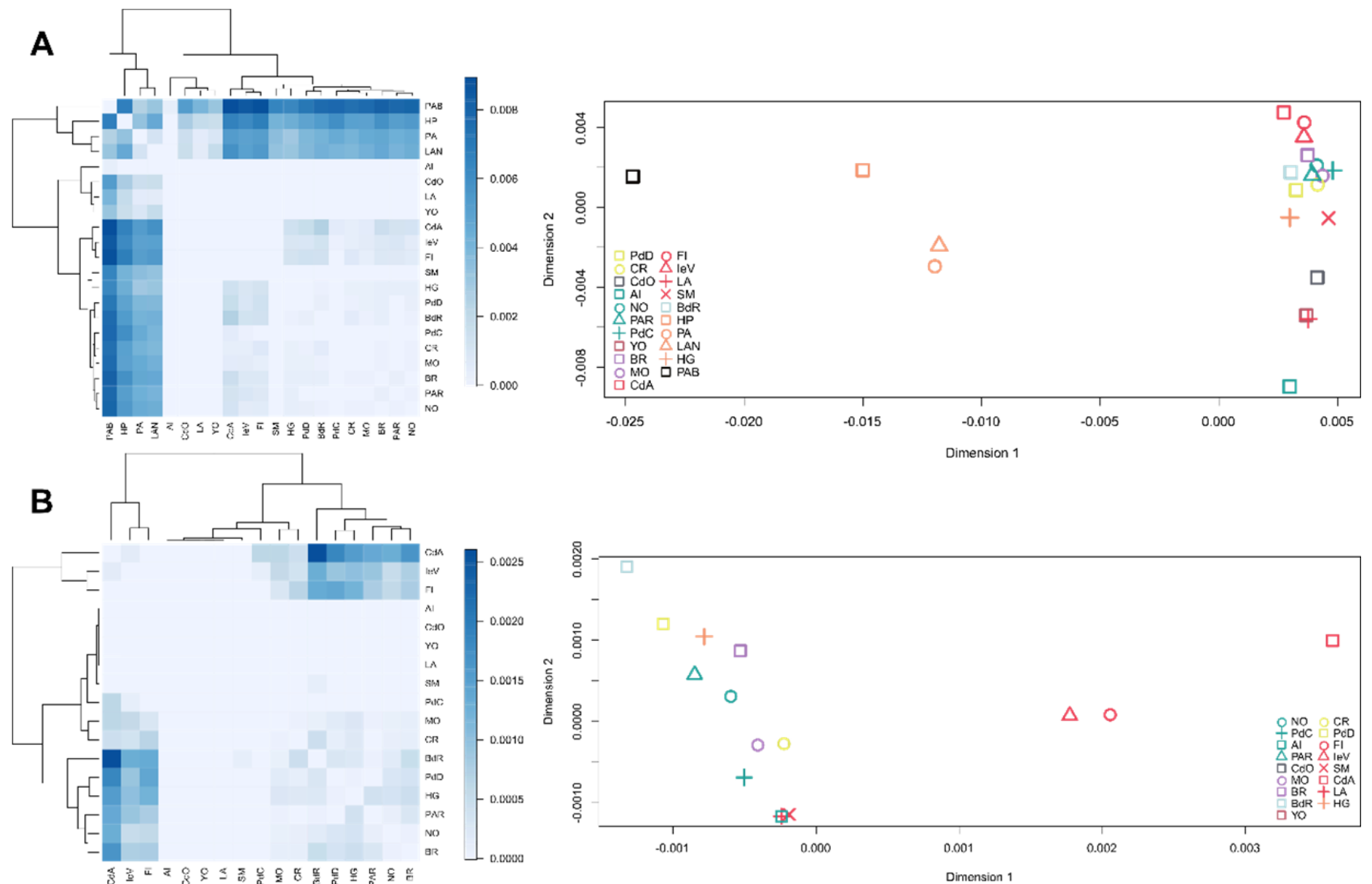

869 Supplementary Figure 6. On the left: heatmap and dendrogram based on $F_{S T}$ matrices A) with the Basque and Gascon samples and B) without them. On the right:

870 Multidimensional scaling (MDS) based on $F_{S T}$ values A) with the Franco-Cantabrian samples and B) without them. 

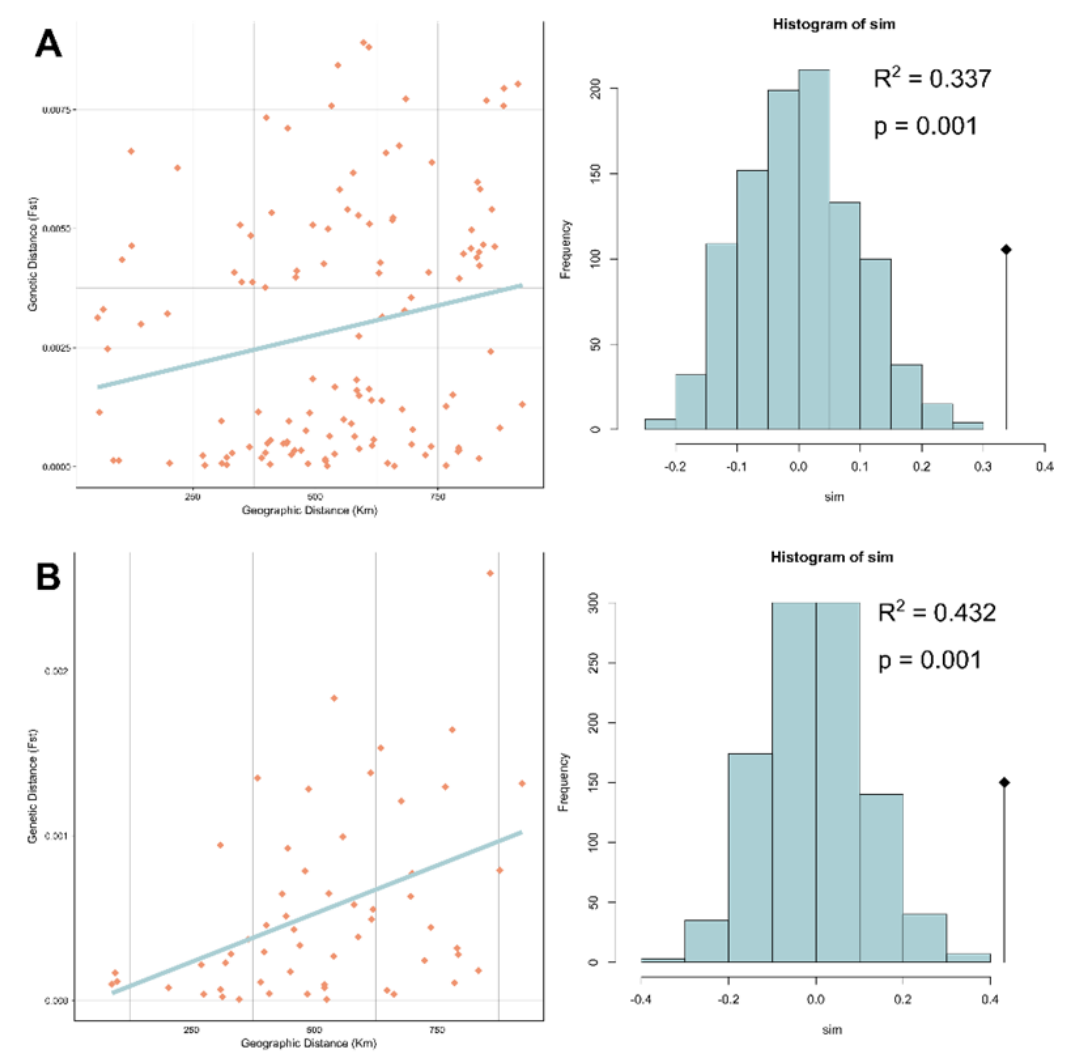

871 Supplementary Figure 7. Mantel test of isolation by distance between the genetic $\left(F_{S T}\right)$ and geographic

872 (in $\mathrm{Km}$ ) distances $\mathbf{A}$ ) with the Basque and Gascon samples and $\mathbf{B}$ ) without them. $\mathrm{R}^{2}$ scores and p-values 873 are within each figure. 


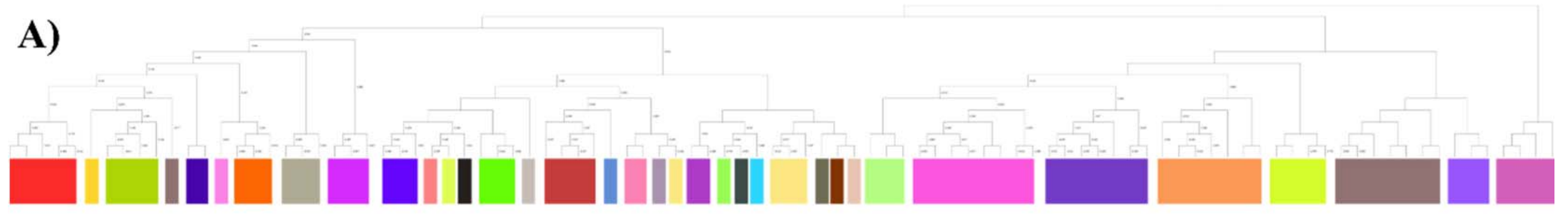

B)

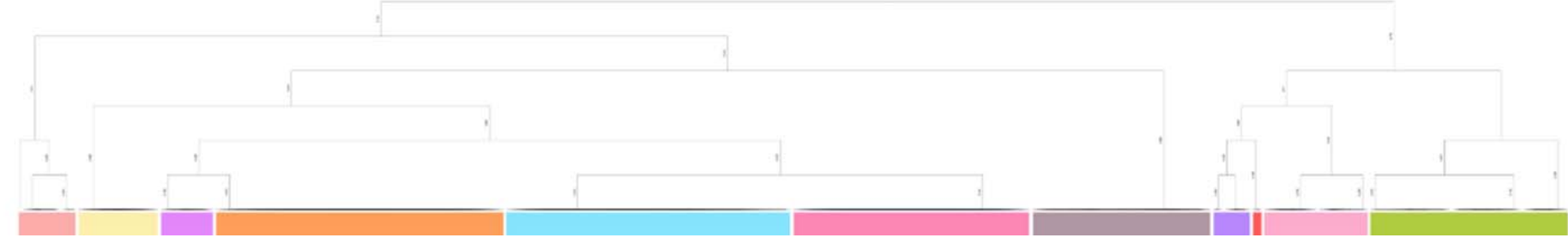



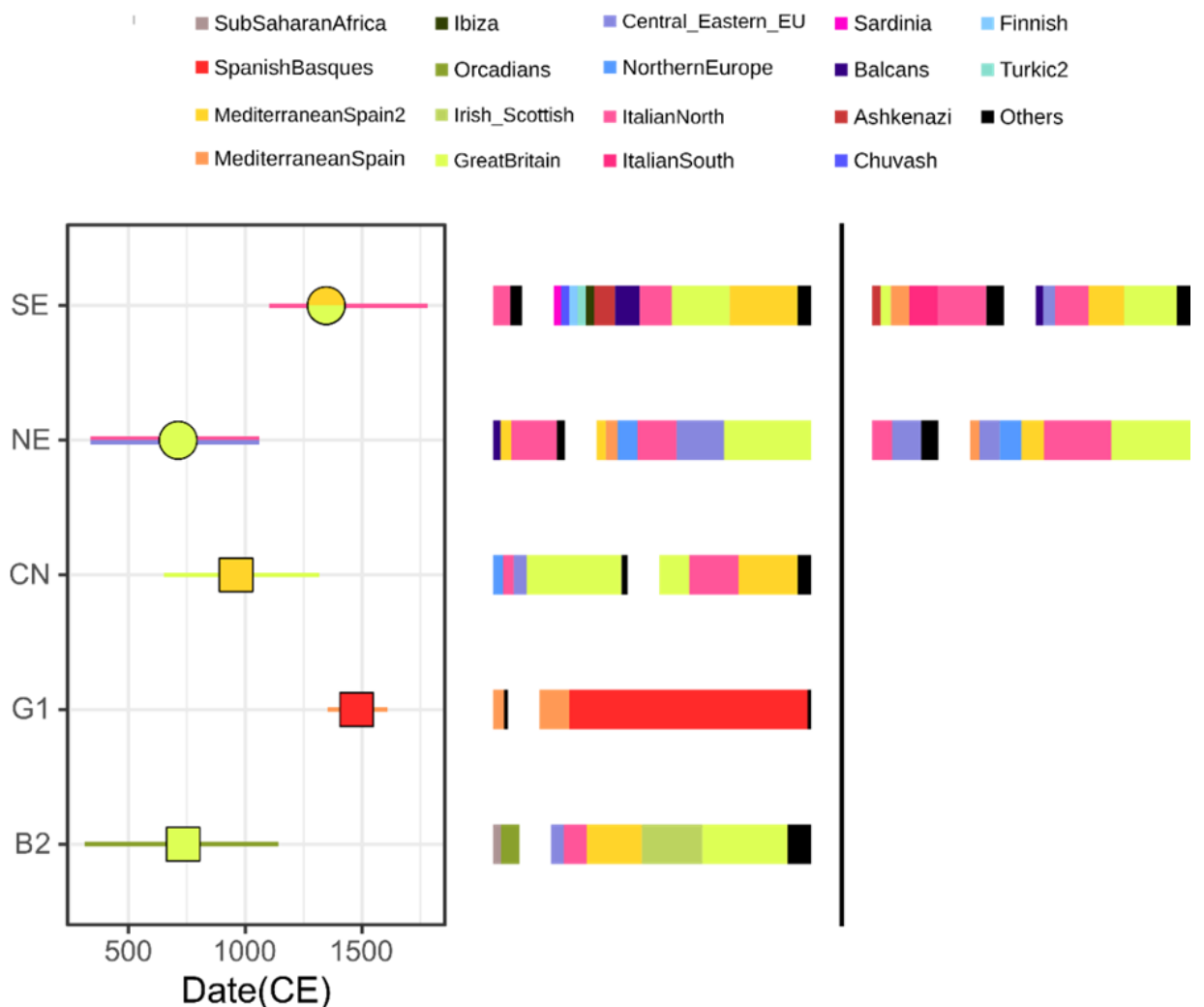

Supplementary Figure 9. Dating results for 5 French targets according to the M analysis in GLOBETROTTER. In the left panel, squares refer to one-date, circles to onedate-multiway. The internal color refers to the highest surrogate's value of the major source, while the color of the CI bars corresponds to the highest surrogate's value of the minor source. Sources are represented as horizontal bars on the right side and are separated by a white space (together the sources account for the $100 \%$ of the values). In the one-date-multiway cases, two different sets of sources are presented and, where needed, both colors are represented for major and minor sources. Dates have been calculated as $1950-(\mathrm{g} * \mathrm{~N})$ where $\mathrm{g}=28$ years and $\mathrm{N}$ is the calculated number of generations in the GLOBETROTTER analysis. 
bioRxiv preprint doi: https://doi.org/10.1101/718098; this version posted October 28, 2019. The copyright holder for this preprint (which was not certified by peer review) is the author/funder, who has granted bioRxiv a license to display the preprint in perpetuity. It is made available under aCC-BY-NC-ND 4.0 International license.
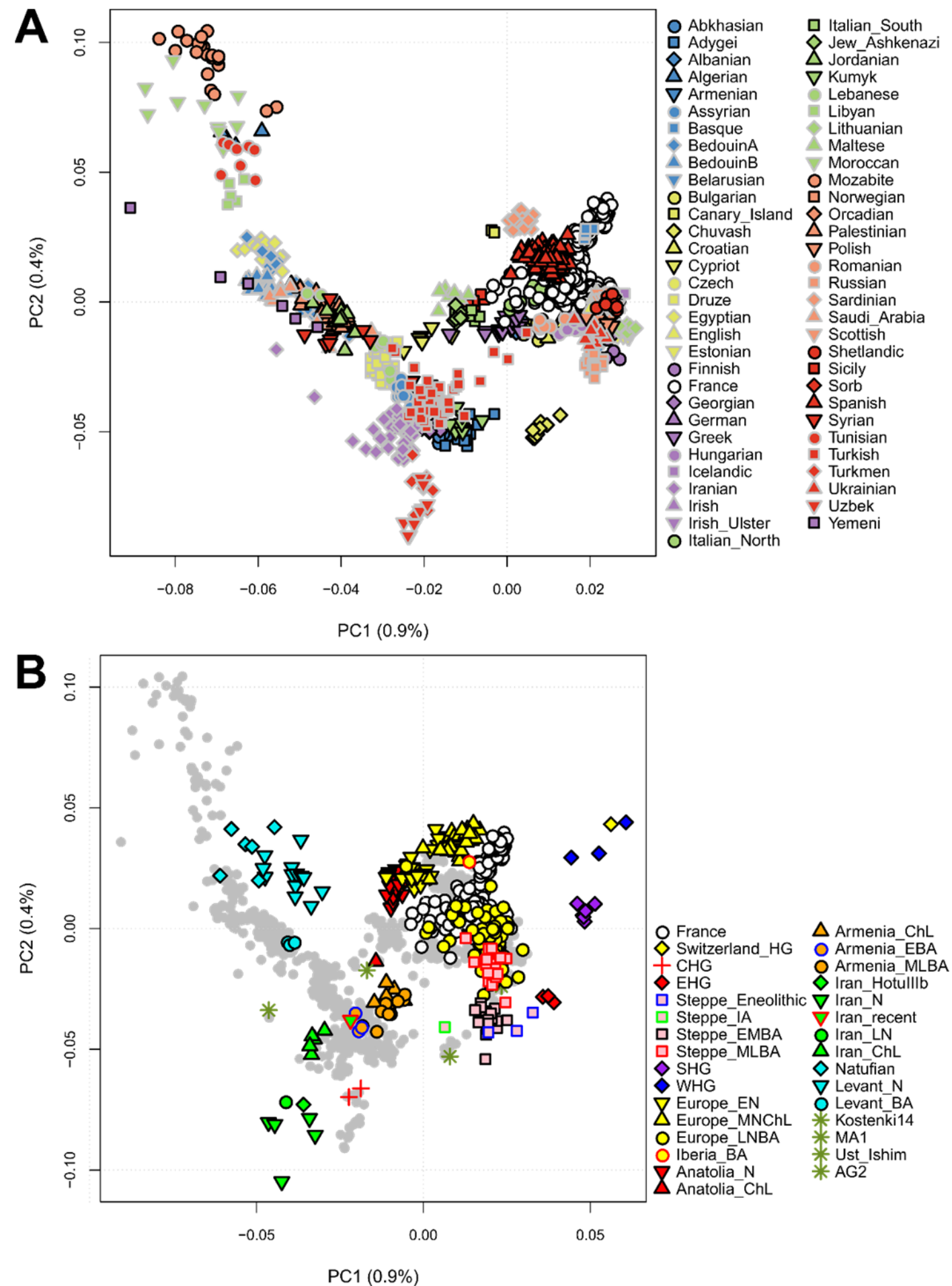

Supplementary Figure 10. Principal component analysis with dataset D. A) Only modern samples; B)

Projection of ancestral populations from different periods on top of the modern samples (grey dots; among the modern populations, only France is distinguishable as white circles). 

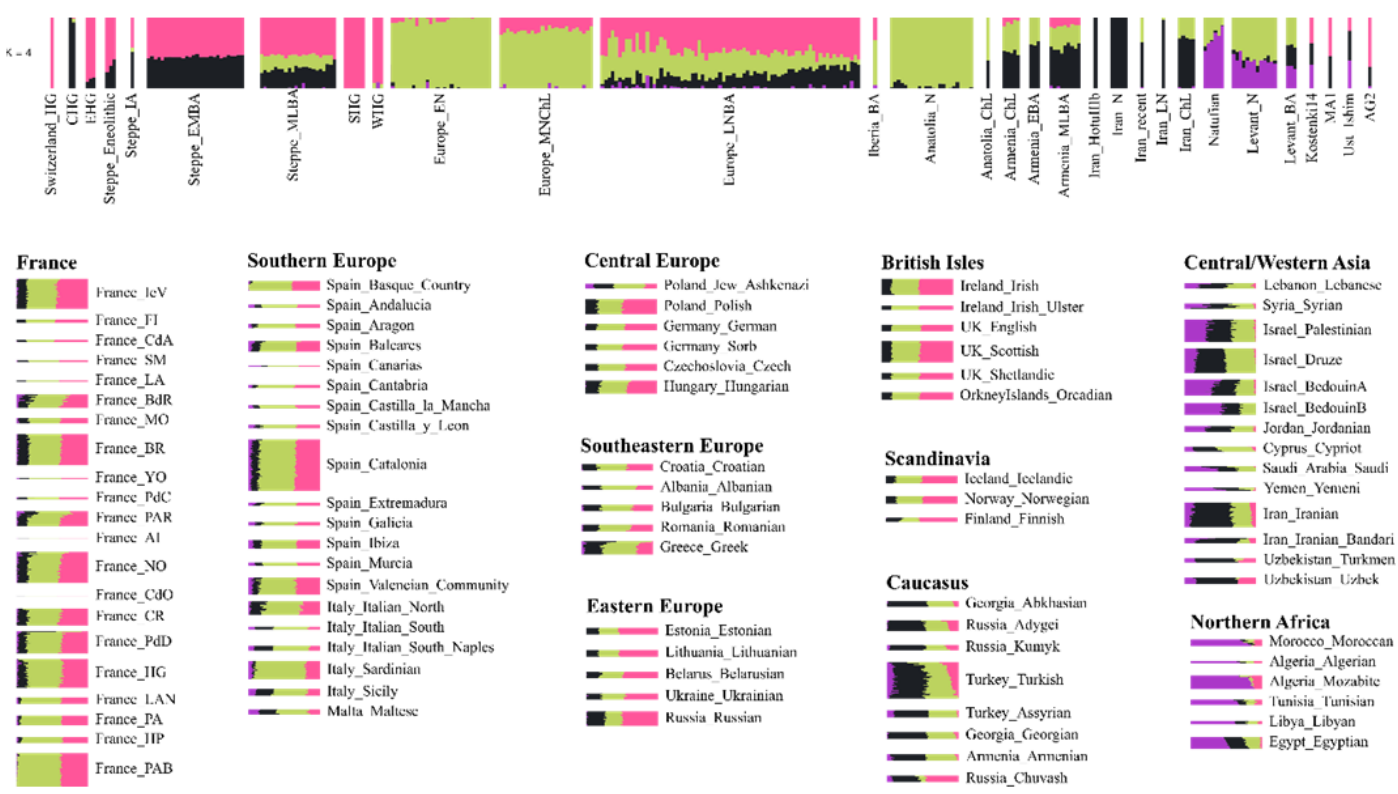

Supplementary Figure 11. ADMIXTURE results for $\mathrm{K}=4$ ancestral components using dataset $\mathrm{D}$. Results for the ancient samples are on the top of the figure. Below, modern samples are organized according to major geographical groupings. 


$\begin{array}{llll}\text { Dataset Samples } & \text { Analysis } & N^{\circ} \text { of SNPs } \\ \text { A } & \mathbf{3 9 5} & \text { Allele frequency / Haplotype-based } & 142,803 / 343,884 \\ \text { B } & \mathbf{7 2 8}(395+333) & \text { Allele frequency } & 154,889 \\ \text { C } & 1527(728+799) & \text { Haplotype-based } & 380,697 \\ \text { D } & 1687(1527-122+282) & \text { Allele frequency } & 163,631\end{array}$

880 Supplementary Table 1. Summary of the dataset composition; both number of samples and number of

881 variants are reported according to the analysis the dataset was used for.

882 